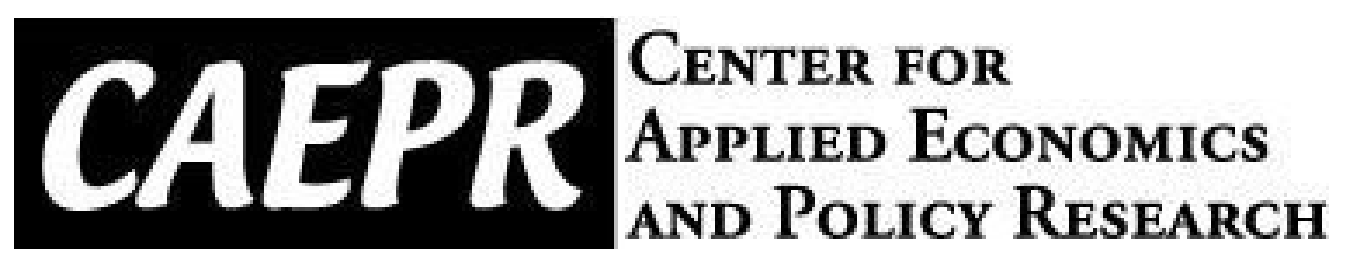

CAEPR Working Paper

\#2015-๑०9

\title{
Identifying Multiple Marginal Effects with a Single Binary Instrument or by Regression Discontinuity
}

\author{
Carolina Caetano \\ University of Rochester \\ Juan Carlos Escanciano \\ Indiana University
}

May 25, 2015

This paper can be downloaded without charge from the Social Science Research Network electronic library at http://papers.ssrn.com/sol3/papers.cfm?abstract_id=2615846

The Center for Applied Economics and Policy Research resides in the Department of Economics at Indiana University Bloomington. CAEPR can be found on the Internet at:

http://www.indiana.edu/ -caepr. CAEPR can be reached via email at caepr@indiana.edu or via phone at 812-855-4050.

(C)2015 by Carolina Caetano and Juan Carlos Escanciano. All rights reserved. Short sections of text, not to exceed two paragraphs, may be quoted without explicit permission provided that full credit, including (c) notice, is given to the source. 


\title{
Identifying Multiple Marginal Effects with a Single Binary Instrument or by Regression Discontinuity*
}

\author{
Carolina Caetano ${ }^{\dagger}$ \\ University of Rochester
}

\author{
Juan Carlos Escanciano ${ }^{\ddagger}$ \\ Indiana University
}

This draft: May 25, 2015.

\begin{abstract}
This paper proposes a new strategy for the identification of all the marginal effects of an endogenous multi-valued variable (which can be continuous, or a vector) in a regression model with one binary instrumental variable. The unobservables must be separable from the endogenous variable of interest in the model. Identification is achieved by exploiting heterogeneity of the "first stage" in covariates. The covariates themselves may be endogenous, and their endogeneity does not need to be modeled. With some modifications, the identification strategy is extended to the Regression Discontinuity Design (RDD) with multi-valued endogenous variables, thereby showing that adding covariates in RDD may improve identification. This paper also provides parametric, semiparametric and nonparametric estimators based on the identification strategy, discusses their asymptotic properties, and shows that the estimators have satisfactory performance in moderate samples sizes. All the proposed estimators can be implemented as Two-Stage Least Squares (TSLS). Finally, we apply our methods to the problem of estimating the effect of air quality on house prices, based on Chay and Greenstone (2005).
\end{abstract}

Keywords: Conditional Instrumental Variables; Endogeneity; Binary Instrument; Regression Discontinuity Design; Varying Coefficients; Nonparametric.

JEL classification: C13; C14; C21; D24

${ }^{*}$ We would like to thank Gregorio Caetano and Kenneth Chay for helpful discussions. We would also like to thank seminar participants at Boston College, Boston University, Harvard-MIT, Northwestern and University of Michigan for useful comments.

${ }^{\dagger}$ Department of Economics, University of Rochester, 238 Harkness Hall, P.O. Box: 270156, Rochester, NY 14627-0156, USA. E-mail: carol.caetano@rochester.edu. Web Page: http://www.carolinacaetano.net/.

${ }^{\ddagger}$ Department of Economics, Indiana University, 105 Wylie Hall, 100 South Woodlawn Avenue, Bloomington, IN 474057104, USA. E-mail: jescanci@indiana.edu. Web Page: http://mypage.iu.edu/ jescanci/. Research funded by the Spanish Plan Nacional de I+D+I, reference number ECO2012-33053. 


\section{Introduction}

Instrumental variables (IV) methods are well established as one of the most useful approaches to identify causal effects in econometric models. Consider the regression model

$$
Y=g(X)+\varepsilon
$$

where $Y$ is the dependent variable, $X$ is endogenous, in the sense that $\mathbb{E}[\varepsilon \mid X] \neq 0$ with positive probability, and $\varepsilon$ is an unobservable error term.

In order to identify the structural regression function $g(\cdot)$ the typical IV identification strategy requires the existence of an instrument $T$ satisfying a validity condition: (i) $T$ is exogenous in the sense that $\mathbb{E}[\varepsilon \mid T]=0$ almost surely (a.s.); and a relevance condition: (ii) the dependence between $X$ and $T$ is "strong enough", so we can uniquely solve $g$ from the equation $\mathbb{E}[Y \mid T]=\mathbb{E}[g(X) \mid T]$.

The relevance condition (ii) imposes severe restrictions on the "complexity" of $T$ depending on the situation (see Newey and Powell (2003)). For instance, if $X$ is continuous and we wish to identify $g$ nonparametrically, then $T$ must be continuous. If $X$ is discrete with $q$ points of support and we wish to identify all of its marginal effects, then $T$ needs to have at least $q$ points of support. If $X$ is a vector of continuous variables, then $T$ must have at least as many components as $X$, even if we impose restrictive restrictions on the shape of $g$, such as linearity.

In this paper we propose a strategy for the identification of $g$ in equation (1) (up to a constant) which applies to cases in which the support of $X$ is larger than that of $T$ and the traditional IV's identification fails. Our validity condition assumes that the instrument is mean independent of the error term conditional on a covariate $Z(\mathbb{E}[\varepsilon \mid T, Z]=\mathbb{E}[\varepsilon \mid Z])$, which may be endogenous. ${ }^{1}$ Note that this validity condition allows $\varepsilon$ to have a non-zero mean and to include functions of $Z$, so $Z$ is not excluded from (1). Our relevance condition requires that we can uniquely solve $g$ (up to a constant) from the equation $\operatorname{Cov}(Y, T \mid Z)=\operatorname{Cov}(g(X), T \mid Z)$. This condition does not require that $T$ be at least as complex as $X$. For simplicity we focus on the case in which $T$ is a binary variable, say $T \in\{0,1\}$, while $X$ takes 3 or more values, and may even be continuous, or a vector. In particular, our approach opens up the possibility of the identification of all the marginal effects of a complex variable $X$ in cases where the instrument may be an experiment or a natural experiment.

Furthermore, when $X$ is continuous, or discrete with more than two points of support, or a vector, the state of the art in the Regression Discontinuity Design (RDD) literature cannot identify $X$ 's marginal effects (see van der Klaauw (2008), Imbens and Lemieux (2008), Lee and Lemieux (2010), and Dinardo and Lee (2011) for surveys of the RDD literature, and Hahn, Todd and van der Klaauw (1990) for an analysis of identification of the classic RDD.) However, we show that since we can think of the RDD as a "local version" of the binary instrumental variable case, we can apply our methodology to the RDD (with a few adaptations) and achieve the identification of $X$ 's marginal effects.

Our identification strategy is based on the observation that if the population is categorized according to a covariate $Z$, the variation that $T$ induces on the distribution of $X$ may be different for different

\footnotetext{
${ }^{1}$ An instrument satisfying our validity condition is referred to as a Conditional IV (CIV) in the literature, see, e.g. Frolich (2007) and Kasy (2009), among others.
} 
values of $Z$. This may allow us to recover a rich (e.g continuous) set of marginal effects. The following example illustrates some of the main ideas.

Example 1.1 Suppose that $X=\left(X_{1}, X_{2}\right)$ and $g$ is linear, so the model in (1) is

$$
Y=\beta_{0}+\beta_{1} X_{1}+\beta_{2} X_{2}+\varepsilon
$$

where $\mathbb{E}\left[\varepsilon \mid X_{1}, X_{2}\right] \neq 0$. Standard $I V$ methods are unable to identify $\beta_{1}$ and $\beta_{2}$ with a single binary instrument $T$, because the classical order condition fails.

For our approach, we require that $T$ be excluded from the structural equation conditional on a variable $Z(\mathbb{E}[\varepsilon \mid Z, T]=\mathbb{E}[\varepsilon \mid Z])$, but $Z$ itself may be endogenous. Then

$$
\begin{aligned}
\mathbb{E}[Y \mid T=1, Z]-\mathbb{E}[Y \mid T=0, Z] & =\beta_{1}\left[\mathbb{E}\left[X_{1} \mid T=1, Z\right]-\mathbb{E}\left[X_{1} \mid T=0, Z\right]\right] \\
& +\beta_{2}\left[\mathbb{E}\left[X_{2} \mid T=1, Z\right]-\mathbb{E}\left[X_{2} \mid T=0, Z\right]\right]
\end{aligned}
$$

To identify $\beta_{1}$ and $\beta_{2}$ we need to invert this equation. The condition that guarantees that we can do this is the following: there exists no pair $\left(\lambda_{1}, \lambda_{2}\right) \neq(0,0)$ such that

$$
\lambda_{1}\left(\mathbb{E}\left[X_{1} \mid T=1, Z\right]-\mathbb{E}\left[X_{1} \mid T=0, Z\right]\right)+\lambda_{2}\left(\mathbb{E}\left[X_{2} \mid T=1, Z\right]-\mathbb{E}\left[X_{2} \mid T=0, Z\right]\right)=0 \quad \text { a.s. }
$$

This condition states that the "first stage" effects of $T$ on $X_{1}$ and $X_{2}$ vary (in a linearly independent manner) with $Z$. Our identification strategy exploits the heterogeneity in the "first stages" to separate the marginal effects of $X_{1}$ and $X_{2}$.

Based on our identification results, we propose parametric, semiparametric and nonparametric estimators of $g$ (up to a constant). In models that are linear in parameters we show that our identification strategy in the binary IV can be implemented with a simple Two-Stage Least Squares (TSLS) estimator that treats the possibly endogenous covariate $Z$ as if it were exogenous and uses interaction terms between $Z$ and $T$ as instruments. We provide Monte Carlo evidence supporting that the TSLS estimator possesses certain robustness properties to the failure of the linear specification of the first stage when the structural equation is correctly specified. For the RDD, we consider a semiparametric varying coefficients specification, which also leads to a simple TSLS implementation and provides a practically convenient way to incorporate covariates' heterogeneity in applications, while dealing with the "curse of dimensionality" problem present when the dimension of $Z$ is moderate or large, which is often the case in applications. Our identification arguments show that, contrary to conventional wisdom, adding covariates in RDD may improve identification. Additionally, we propose nonparametric estimators that relax the functional form assumptions, and discuss the rates of consistency based on results by Blundell, Chen and Kristensen (2007). In all cases (parametric and nonparametric with binary IV and semiparametric and nonparametric with the RDD) our identification strategy can be implemented with off-the-shelf econometric software.

Our approach has some connections with the local average treatment effect literature (see Imbens and Angrist (1994) and Angrist, Graddy and Imbens (2000)) and the related RDD literature (see Hahn, Todd and van der Klaauw (1990)). However, both literatures have traditionally focused on 
the causal impact of a binary endogenous variable and discrete or continuous instrument, whereas here we are interested in continuous endogenous variables and a binary instrument. Our results also complement alternative identification strategies for binary instruments and continuous endogenous variables in Chesher (2003), D'Haultfoeuille and Fevrier (2014), Torgovitsky (2014), D'Haultfoeuille, Hoderlein and Sasaki (2013) and Masten and Torgovitsky (2014). None of these papers exploit the heterogeneity of the "first stage" conditional on possibly endogenous covariates.

The rest of the paper is organized as follows. Section 2 develops an example in detail which shows explicitly what are the fundamental requirements of our method, as well as why it works. Section 3 focuses on the binary IV case. It presents the identification results and also shows that the identification strategy can be implemented with a suitable TSLS estimator. Section 4 focuses on the RDD case. It extends the identification results of the binary IV case to the RDD, and it also proposes a TSLS estimator for a semiparametric varying coefficient specification. We discuss implementation in the fully non-parametric cases both in the binary IV case as well as in the RDD in the Appendix A.2. Section 5 reports the results of Monte Carlo experiments. Section 6 contains an empirical application of our method to the problem of estimating the effect of air quality on house prices, based on Chay and Greenstone (2005). This empirical example is particularly covenient because we are able to explore both a standard binary IV as well as an RDD design in the same problem, and using the same data. We conclude in Section 7.

\section{An Example}

The idea is best explained in an applied example. We will consider the problem of estimating the marginal effect of the amount a woman smokes during pregnancy (average daily number of cigarettes) on the baby's weight at birth (see Almond and Currie (2011) for a survey of the literature on this problem.) The variable of interest, "smoking," is naturally prone to endogeneity, given that there are many pre-existing selection factors associated both with smoking and with birth weight. Examples of such factors include the mother's education level, marital status, age, etc. There is a notorious shortage of instruments for smoking. Existing options explore cigarette tax changes or variations across localities, but these instruments tend to be weak, meaning that pregnant women tend to change their smoking behavior very little because of the changes in cigarette prices. Experimental approaches fare better, with the intervention causing smoking cessation on average in $6 \%$ of the population (see Lumley et al. (2011)). However, experiments provide only a binary instrument, and so it is impossible to uncover the effects of each marginal cigarette in the standard approach.

The following setup is entirely fictitious, but we believe that the association of our notation to a real problem can be helpful. Although the exposition below is rather informal, the rigorous arguments behind all claims can be seen in Example 3.1 in Section 3. In the maternal smoking context, the variable $X$ represents the average number of cigarettes smoked per day during pregnancy, which may take several values. Suppose that an experiment implements an intervention in the treatment group that incentivizes women to reduce, and hopefully quit smoking, while the women in the control group are merely observed. $T$ is thus equal to one if the woman is in the treatment group, and zero otherwise. 
If we were able to observe the same woman under treatment and control, we could use the variation in the smoking dosages across the different women to recover the entire response function of birth weight to smoking. Precisely, we would learn the effect of the first cigarette from the women who smoked one cigarette, and then quit after the intervention. We would learn the effect of the second cigarette both from the women that smoked 2 cigarettes and then reduced to 1 cigarette under the intervention, or by subtracting the effect of the first cigarette from the effect found from the women that smoked 2 cigarettes and then quit after the intervention. We could continue with this strategy until we had the effects of each subsequent cigarette.

Unfortunately, experimental data does not allow us to observe the same woman under both treatment and control. The main insight of our method is that we can sometimes use a covariate to classify women into different groups, and thus generate artificially heterogeneous counterfactuals. For example, suppose that $Z$ is the number of years of education of the mother. Although the experiment may have been performed without taking education into consideration at all, we can still separate women according to their education level, as long as this information is included in our data. The following table proposes a fictitious situation in which we do just this. Column (I) represents the years of education,

\section{Table 1: Identification Idea}

\begin{tabular}{c|ccc|ccc|ccc|c}
\hline \hline$(\mathrm{I})$ & $(\mathrm{II})$ & $(\mathrm{III})$ & $(\mathrm{IV})$ & $(\mathrm{V})$ & $(\mathrm{VI})$ & $(\mathrm{VII})$ & $(\mathrm{VIII})$ & $(\mathrm{IX})$ & $(\mathrm{X}) *(\mathrm{XI})$ & \\
$\mathbf{Z}$ & $\overline{\mathbf{X}}_{\mathbf{0 , \mathbf { Z }}}$ & $\overline{\mathbf{X}}_{\mathbf{1}, \mathbf{Z}}$ & $\boldsymbol{\Delta}_{\mathbf{Y}}(\mathbf{Z})$ & $\mathbb{P}_{\mathbf{0}, \mathbf{Z}}(\mathbf{0})$ & $\mathbb{P}_{\mathbf{0}, \mathbf{Z}}(\mathbf{1})$ & $\mathbb{P}_{\mathbf{0}, \mathbf{Z}}(\mathbf{3})$ & $\mathbb{P}_{\mathbf{1}, \mathbf{Z}}(\mathbf{0})$ & $\mathbb{P}_{\mathbf{1}, \mathbf{Z}}(\mathbf{1})$ & $\mathbb{P}_{\mathbf{1}, \mathbf{Z}}(\mathbf{3})$ & row \# \\
\hline 6 & 3 & 2 & 10 & 0 & 0 & 1 & 0 & $1 / 2$ & $1 / 2$ & $(1)$ \\
10 & 3 & 2 & 22 & 0 & 0 & 1 & $1 / 5$ & $1 / 5$ & $3 / 5$ & $(2)$ \\
17 & 3 & 2 & 30 & 0 & 0 & 1 & $1 / 3$ & 0 & $2 / 3$ & $(3)$ \\
\hline \hline
\end{tabular}

and at first we are considering only 3 possibilities: 6, 10 and 17. Columns (II) and (III) show the average amount smoked by the women in the control and treatment groups, respectively, for the given number of years of education. Curiously, on average all groups reduced one cigarette because of the intervention. This is not a requirement of our method, we just want to show what can be achieved even when there is on average no variation at all in the "first stages." Column (IV) shows the average difference (in grams) in the birth weight between the treatment and the control groups for that level of education $\left(\Delta_{Y}(Z)=\mathbb{E}[Y \mid T=1, Z]-\mathbb{E}[Y \mid T=0, Z]\right)$.

The first fundamental assumption of our method is a validity condition, which requires that $\mathbb{E}[\varepsilon \mid T, Z]=\mathbb{E}[\varepsilon \mid Z]$. It implies that within each education level we can compare treatment and control groups to obtain the causal effect of the intervention for each of the education groups $\left(\Delta_{Y}(Z)=\right.$ $\mathbb{E}[g(X) \mid T=1, Z]-\mathbb{E}[g(X) \mid T=0, Z])$. In this example, the smoking variable $X$ takes the values 0 , 1 or 3 (generalization to a more complex $X$ will become clear later). In this multi-valued setting we cannot translate the effect of the intervention directly into information about the actual causal effects of smoking on birth weight, which is described by the function $g$, because the treatment and control 
groups for a given education level are only comparable as a whole.

We do, however, observe exactly what each woman consumed. Columns (V) to (VII) show the smoking distribution in the control group. The numbers in row (1) are the fractions of the women educated 6 years and in the control group that smoked 0,1 or 3 cigarettes, respectively. In this example everyone in the control group smokes 3 cigarettes (one can think of an experiment that specifically recruited women who smoked 3 cigarettes to participate). This is also not a requirement of the method, but it simplifies the explanations to have one less moving part.

Columns (VIII) to (X) show the corresponding fractions in the treatment group. As we can see, each row is different. It means that the intervention did not affect all groups in the same way. Even though each education group had the same reaction to the intervention on average, a reduction of one cigarette, the distribution of behaviors is very different. Among the women educated 6 years, half reduced their smoking by 2 cigarettes, while the other half did not modify their behavior. The women educated 10 years had more divided reactions, with $3 / 5$ keeping their old habit, while the remaining were divided, half quitting, and half reducing to one cigarette per day. Among the women educated 17 years, an even higher fraction did not modify their behavior, 2/3, but the ones that did all quit. The resulting distributions of smoking levels across the different education levels are very varied. It is this variation in the distributions that is at the heart of our approach. It does not matter that all the average effects are the same, it would not even matter if there were no effects on average at all. Our ability to identify the marginal effects comes from the fact that the instrument affected the distribution of $X$ differently across the different $Z$, as we will show below.

This variation in the distributions also explains the outcome differences seen on column (IV). Even though the women behaved the same on average, if the effects are nonlinear the reduced effects across the groups can vary. For example, if the birth weight is affected disproportionately more by the first cigarette than the rest, we may find that the group in row (3), where a higher proportion of women quit, may have a much more pronounced effect in the birth weight than the group in row (1), where nobody quit. It is important to notice that the different numbers in column (IV) could also be explained by a non-separability of $X$ and $Z$ in the structural equation, for example if smoking could interact with education in such a way that a reduction of one cigarette was more effective among women educated 10 years than among women educated 6 years. This explanation is, however, ruled out indirectly by the separability between the error term $\varepsilon$ and $X$ in the model in (1) together with the validity condition. We can thus write $\mathbb{E}[Y \mid T=1, Z]-\mathbb{E}[Y \mid T=1, Z]=\mathbb{E}[g(X) \mid T=1, Z]-\mathbb{E}[g(X) \mid T=0, Z]$. Therefore any differences across the rows of column (IV) must be due to nonlinearities of $g$ combined with differences in the distribution of $X$ across the different levels of education.

We will use the variations in distributions in the following manner: the treatment and control groups are comparable for each level of education, but only as a whole. Hence, the differences in the outcomes between treatment and control are the result of the effects of the possible smoking behaviors combined with the differences in the probabilities of each smoking behavior. For example, row (1) gives us $10=\mathbb{E}[Y \mid T=1, Z=6]-\mathbb{E}[Y \mid T=0, Z=6]=0 \cdot g(0)+0.5 \cdot g(1)+0.5 \cdot g(3)-[0 \cdot g(0)+0 \cdot g(1)+1 \cdot g(3)]=$ $0.5 \cdot g(1)-0.5 \cdot g(3)$. The resulting equation provides some information about $g$, but combining this 
with the differences for other $Z$ 's, we can get a system of equations:

$$
\begin{aligned}
0.5 g(1)-0.5 g(3) & =10 \\
0.2 g(0)+0.2 g(1)-0.4 g(3) & =22 \\
0.33 g(0)-0.33 g(3) & =30
\end{aligned}
$$

Notice that in order to combine the information learned across all the different levels of $Z$ into a common system, it is imperative that $Z$ 's own effect be separable from that of $X$. The separability of the model is thus not a simplification, it is vital for the method.

This system of equations has 3 equations and 3 unknowns. However, only two equations are linearly independent (since $0.4(2)+0.6(4)=(3)$ ). In fact, if we had used more values of the variable $Z$, we could have more equations, but it would not change the fact that at most two equations would be independent. This is caused by the fact that the coefficients of each of these equations always add up to zero, as can be easily verified in the example above. The reason for this phenomenon is that the coefficients come from the subtraction of probabilities. Since probabilities always add up to one, the subtraction of two sets of probabilities always adds up to zero. This is true not only for this example, but for all cases. For example, if $X$ assumes $q$ values, then the maximum number of linearly independent equations we can get is $q-1$.

Since we have 2 linearly independent equations, we cannot recover the values of $g(0), g(1)$, and $g(3)$, but we can recover the value of any increment. It is straightforward to see in this example that, from equation (2), $g(3)-g(1)=-20$, from equation (4), $g(3)-g(0)=-90$, and combining both results, $g(1)-g(0)=-70$. In a situation where $X$ assumes more values, say $q$, we can get all the increments provided we have $q-1$ linearly independent equations. Hence, the second fundamental requirement of this method is a relevance condition which requires that the change in the distribution of behaviors between treatment and control groups differs for the $Z$ 's. If $X$ takes $q$ values, there must be enough variation for at least $q-1$ values of $Z$ (indirectly it requires that $Z$ must assume at least $q-1$ values).

We never explained why would such variations in the change of distributions occur. This depends on the example, the particular intervention, and the chosen $Z$. For example, suppose that the intervention in our example requires that the women in the treatment group read extensive material. This intervention could affect women of different levels of education in different manners. Less educated women might be less likely to get through the material, and therefore to quit as a result of the intervention. In the fictitious example we created the more educated women were indeed the most likely to quit, even if on average the behaviors were the same across all levels of education. In a real example it is unlikely that even the average behavior will be the same across all groups, thus possibly generating even more variation. 


\section{The Case with a Binary Instrumental Variable}

\subsection{Identification}

We observe $(Y, X, Z, T)$ satisfying the model

$$
Y=g(X)+\varepsilon
$$

where the following exclusion restriction holds

Assumption 1 (validity) $\mathbb{E}[\varepsilon \mid Z, T]=\mathbb{E}[\varepsilon \mid Z]$ a.s.

We can thus write

$$
\mathbb{E}[Y \mid Z, T=1]-\mathbb{E}[Y \mid Z, T=0]=\mathbb{E}[g(X) \mid Z, T=1]-\mathbb{E}[g(X) \mid Z, T=0] \text { a.s. }
$$

Identifying $g$ (up to location) from this implicit equation depends on our ability to invert it. To better understand the conditions that guarantee the invertibility of equation (6) consider first the following example for the case where $X$ and $Z$ are discrete. This example formalizes the discussion in Section 2 , and it extends naturally to the general case.

Example 3.1 ( $X$ and $Z$ discrete) Denote by $\mathcal{S}_{X}:=\left\{x_{1}, \ldots, x_{q}\right\}$ and $\mathcal{S}_{Z}:=\left\{z_{1}, \ldots, z_{l}\right\}$ the supports of the distributions of $X$ and $Z$, respectively, with $q<\infty$ and $l<\infty$. The classic nonparametric $I V$ identification strategy is based on the equation

$$
\mathbb{E}[Y \mid T]=\mathbb{E}[g(X) \mid T]
$$

which in this case translates into the system of equations $\mathbf{r}=P \mathbf{g}$, where $\mathbf{r}:=(\mathbb{E}[Y \mid T=0], \mathbb{E}[Y \mid T=1])^{\prime}$, $P=\left(p_{t j}\right)$ is the $2 \times q$ matrix with entries $p_{t j}:=\mathbb{P}\left(X=x_{j} \mid T=t\right), t=0,1, j=1, \ldots, q$, and $\mathrm{g}:=\left(g\left(x_{1}\right), \ldots, g\left(x_{q}\right)\right)^{\prime}\left(A^{\prime}\right.$ denotes the transpose of $\left.A\right)$. In this classic setting the matrix $P$ has a rank of at most 2, and so $g$ is not identified if $q>2$ (see Newey and Powell (2003)). In fact, we can only identify linear functionals c' $\mathbf{g}$ where $c$ is spanned by the two rows of P (see Severini and Tripathi (2006, 2012)), which are not necessarily of interest.

Our identification strategy consists of inverting equation (6), which in this context can be written as $\mathbf{m}=A \mathbf{g}$, where, $\mathbf{m}:=\left(m\left(z_{1}\right), \ldots, m\left(z_{l}\right)\right)^{\prime}$, with $m(z):=\mathbb{E}[Y \mid T=1, Z=z]-\mathbb{E}[Y \mid T=0, Z=z]$, $z \in \mathcal{S}_{Z}$, and the matrix $A$ is given by $P_{1}-P_{0}$, where $P_{t}=\left(p_{t i j}\right)$ is the $l \times q$ matrix with entries $p_{t i j}=\mathbb{P}\left[X=x_{j} \mid T=t, Z=z_{i}\right], i=1, \ldots, l, j=1, \ldots, q$ and $t=0,1$. Notice that since $P_{0}$ and $P_{1}$ are matrices of probabilities, $A \iota=0$, where $\iota$ denotes the $q \times 1$ vector of ones. Therefore, $A$ is not full-rank, and thus $g$ is not identified from (6). However, in this context we can identify linear functionals $c^{\prime} \mathbf{g}$ with $c$ in a space of dimension $\operatorname{rank}(A)$. In particular, if $\operatorname{rank}(A)=q-1$, then all linear functionals $c^{\prime} \mathbf{g}$ with $c^{\prime} \iota=0$ are identified. In this case, all increment effects $g\left(x_{h}\right)-g\left(x_{j}\right), h \neq j$, are identified. Of course, this is only possible if the order condition $l \geq q-1$ holds, so $Z$ needs to assume at least $q-1$ different values. 
This discussion extends to the general case as follows. With some abuse of notation, we write equation (6) also as

$$
m=A g,
$$

where now $A g:=\mathbb{E}[g(X) \mid Z, T=1]-\mathbb{E}[g(X) \mid Z, T=0], A$ is a continuous (i.e. bounded) linear operator, $A: \mathcal{G} \subseteq L_{2}(X) \rightarrow L_{2}(Z)$, where henceforth, for a generic random vector $\zeta, L_{2}(\zeta)$ denotes the Hilbert space of squared-integrable functions with respect to the distribution of $\zeta$. Here $\mathcal{G}$ is a subspace of $L_{2}(X)$ that may incorporate prior restrictions on $g$ such as functional form restrictions or shape restrictions. We introduce our identification assumption as follows. Define $\mathcal{N}(A)=\{g \in \mathcal{G}: A g=0\}$, the null space of $A$. Our fundamental relevance condition requires that the null space of $A$ is composed exclusively of the constant functions:

Assumption 2 (relevance) $\mathcal{N}(A)=\{f \in \mathcal{G}: f \equiv c \in \mathbb{R}\}$.

Notice that the identification condition in Example 3.1 that $\operatorname{rank}(A)=q-1$ is equivalent to Assumption 2 in the discrete support case, $\operatorname{since} \operatorname{dim}(\mathcal{N}(A))+\operatorname{rank}(A)=q$. In the general case, Assumption 2 is a nonparametric rank condition which is the analogue of the $L_{2}$-completeness condition required in nonparametric IV (see Newey and Powell (2003), Blundell, Chen and Kristensen (2007), Andrews (2011) and D'Haultfoeuille (2011) for discussions on completeness). We compare formally these two identification assumptions in Remark 3.5 below.

Theorem 3.1 Under Assumptions 1 and 2, $g$ is identified up to location.

Proof. Note that $A \widetilde{g}=m$, with $\widetilde{g}(X)=g(X)-\mathbb{E}[g(X)]$ and $A$ is invertible on the orthocomplement of $\mathcal{N}(A)$, which by Assumption 2 is given by $\mathcal{N}^{\perp}=\{\lambda \in \mathcal{G}: \mathbb{E}[\lambda(X)]=0\}$. Thus, since $\widetilde{g} \in \mathcal{N}^{\perp}$ it holds that $\widetilde{g}=A^{-1} m$.

Necessary conditions for Assumption 2 in the nonparametric case are that $X$ and $Z$ have the same level of complexity (e.g. both are continuous) and that they are not functionally dependent (separability). Intuitively, what is needed for Assumption 2 to hold is that the differences between the distributions of $X$ under "treatment" $(T=1)$ and "control" $(T=0)$ groups vary sufficiently with $Z$. The following examples show the exact meaning of Assumption 2 in some special cases.

Example 3.2 Suppose that $(X, Z)$ is jointly normal conditionally on T, i.e.

$$
(X, Z) \mid T \sim N\left(\left(\begin{array}{l}
0 \\
0
\end{array}\right),\left(\begin{array}{cc}
1 & \rho_{T} \\
\rho_{T} & 1
\end{array}\right)\right) .
$$

Following Dunker, Florens, Hohage, Johannes and Mammen (2014), we can compute

$$
\mathbb{E}[g(X) \mid Z=z, T=t]=(2 \pi)^{-3 / 4} \exp \left(-\frac{z^{2}}{2}\right) \sum_{j=0}^{\infty} \mu^{j}\left(\rho_{t}\right) \mathbb{E}\left[g(X) p_{j}(X)\right] \frac{z^{j}}{\sqrt{j !}}
$$


where $p_{j}$ are the Hermite functions, $p_{j}(x)=(j ! 2 \pi)^{-1 / 2} \exp \left(-0.5 x^{2}\right) H_{j}(x)$, with $H_{j}$ the $j$-th Hermite polynomial, and $\mu(\rho)=\rho / \sqrt{1-\rho^{2}}$. Therefore,

$$
A g(z)=(2 \pi)^{-3 / 4} \exp \left(-\frac{z^{2}}{2}\right) \sum_{j=0}^{\infty}\left\{\mu^{j}\left(\rho_{1}\right)-\mu^{j}\left(\rho_{0}\right)\right\} \mathbb{E}\left[g(X) p_{j}(X)\right] \frac{z^{j}}{\sqrt{j !}} .
$$

By the completeness of the Hermite polynomials, Assumption 2 in this context translates into $\rho_{1} \neq \rho_{0}$ (with $\left.\mathcal{G}=L_{2}(X)\right)$. Notice that if $f_{X \mid T, Z}$ denotes the density of $X$ conditional on $T$ and $Z$, then

$$
f_{X \mid T=t, Z=z}(x)=\frac{1}{\sqrt{2 \pi\left(1-\rho_{t}^{2}\right)}} \exp \left(-\frac{\left(x-\rho_{t} z\right)^{2}}{2\left(1-\rho_{t}^{2}\right)}\right) .
$$

Therefore, the condition $\rho_{1} \neq \rho_{0}$ guarantees that the difference between the distribution of $X$ between treatment and control groups varies with $Z$.

Example 3.3 (Linear Multivariate Model) Suppose that $g$ is linear, so that the model is

$$
Y=\beta^{\prime} X+\varepsilon, \quad \mathbb{E}[\varepsilon \mid Z, T]=\mathbb{E}[\varepsilon \mid Z],
$$

where $X$ is a d-dimensional vector. Note that $\varepsilon$ is not required to have zero mean, so it may include an intercept and/or functions of $Z$. In this model, the integral equation can be written as

$$
\mathbb{E}[Y \mid Z, T=1]-\mathbb{E}[Y \mid Z, T=0]=\beta^{\prime}(\mathbb{E}[X \mid Z, T=1]-\mathbb{E}[X \mid Z, T=0]),
$$

or in short (using the generic notation $\Delta_{V}=\mathbb{E}[V \mid Z, T=1]-\mathbb{E}[V \mid Z, T=0]$ )

$$
\Delta_{Y}=\beta^{\prime} \Delta_{X}
$$

Hence, in this example $\mathcal{G}=\left\{b^{\prime} X: b \in \mathbb{R}^{d}\right\}$ and Assumption 2 is equivalent to

$$
\mathbb{E}\left[\Delta_{X} \Delta_{X}^{\prime}\right] \text { is positive definite. }
$$

It is straightforward to see why $\beta$ is identifiable under this condition, since from equation (9) $\beta=$ $\left(\mathbb{E}\left[\Delta_{X} \Delta_{X}^{\prime}\right]\right)^{-1} \mathbb{E}\left[\Delta_{X} \Delta_{Y}\right]$. In practice, this condition requires that the "first stages" of the several elements in the vector $X$ vary with $Z$ in a linearly independent manner. Notice that in linear models we can relax the conditions on the complexity of $Z$. For example, even though $X$ is multivariate, $Z$ may be univariate (though it must assume at least d different values).

The linear multivariate model contains interesting examples as special cases. Consider the examples below.

The discrete case: In Example 3.1 we discussed the case where the endogenous variable is discrete. However, it is possible to analyze the discrete case within the framework of a linear model. Suppose that the endogenous variable $D$ has support $\mathcal{S}_{D}:=\left\{d_{1}, \ldots, d_{q}\right\}$, then

$$
\begin{aligned}
g(D) & =g\left(d_{1}\right)+\left(g\left(d_{2}\right)-g\left(d_{1}\right)\right) 1\left(D=d_{2}\right)+\cdots+\left(g\left(d_{q}\right)-g\left(d_{1}\right)\right) 1\left(D=d_{q}\right) \\
& \equiv \alpha+\beta^{\prime} X,
\end{aligned}
$$


where $\alpha=g\left(d_{1}\right), X=\left(1\left(D=d_{2}\right), \ldots, 1\left(D=d_{q}\right)\right)^{\prime}$, and $\beta=\left(g\left(d_{2}\right)-g\left(d_{1}\right), \ldots, g\left(d_{q}\right)-g\left(d_{1}\right)\right)^{\prime}$ denotes the increment effects of interest.

Nonlinear cases: Several nonlinear models cans still be written as linear-in-parameters models, such as, for example, the piecewise linear model. The simplest case is

$$
Y=\beta_{1} D+\beta_{2} D 1(D>0)+\varepsilon
$$

where $D$ is an endogenous variable. In the application Section 6 we discuss connected piecewise linear models with more than two pieces, which can also be modeled similarly. We can also include some models with infinite, but parametric, variation in the marginal effects, such as

$$
Y=\beta_{1} D+\beta_{2} D^{2}+\varepsilon
$$

Classic IV identification conditions require two instruments in all examples above. In contrast, our identification assumption can still be applied when only $T$ is used as instrument. These models, although nonlinear in variables, are linear in parameters, and therefore they can be treated as the linear model (8) above, simply defining $X=(D, D 1(D>0))^{\prime}$ and $X=\left(D, D^{2}\right)^{\prime}$, respectively.

Remark 3.1 (Relation to classical IV with covariates) Although the validity condition $\mathbb{E}[\varepsilon \mid T, Z]=$ $\mathbb{E}[\varepsilon \mid Z]$ in our approach is the same used in the classical IV method with covariates (see Frolich (2007) and Kasy (2009)), in our method it is never necessary to specify how $Z$ enters in the structural equation. That is, we do not need to specify $h(Z):=\mathbb{E}[\varepsilon \mid Z]$. Our method does not identify $h(\cdot)$, which is a nuisance parameter in our setting. In contrast, traditional IV methods try to identify $g$ and $h$ from the moment restrictions

$$
\mathbb{E}[Y-g(X)-h(Z) \mid Z, T]=0 \text { a.s. }
$$

Remark 3.2 (Relation to panel data models) Consider the panel data model

$$
Y_{t}=g\left(X_{t}\right)+\eta+u_{t}
$$

where $\mathbb{E}\left[u_{t} \mid X_{0}, X_{1}\right]=0$. Then,

$$
Y_{1}-Y_{0}=g\left(X_{1}\right)-g\left(X_{0}\right)+u_{1}-u_{0}
$$

and we can identify $g$ up to a constant from $\mathbb{E}\left[Y_{1}-Y_{0} \mid X_{0}, X_{1}\right]$. We can understand this heuristically as an $I V$ model with $T=t$ as the IV. Even though the $I V$ is binary, we can identify $g$ 's derivatives in this case because we observe the counterfactuals $X_{0}$ and $X_{1}$ for the same observation. In the standard $I V$ setting we cannot observe the counterfactuals. Our idea is to generate artificial counterfactuals by classifying observations according to a covariate $Z$ so that we can apply a similar strategy.

Remark 3.3 (Choosing $Z$ ) The classification variables $Z$ are chosen by the researcher, and often there can be many options. The main consideration in the choice of $Z$ is that it must be separable from $X$ in the structural equation. It is important to notice that not all covariates in the model need to be separable 
from $X$, just those which will be used as $Z$ in our identification approach. To better understand how to make this choice, consider an extended model

$$
Y=g\left(X, Z_{c}\right)+\varepsilon
$$

where $\mathbb{E}\left[\varepsilon \mid T, Z_{c}, Z\right]=\mathbb{E}[\varepsilon \mid Z]$. In this model the researcher chose to separate the variables into two groups. The variables $Z_{c}$ are included into the model as exogenous controls. The variables $Z$ are used as the classification variable. Notice that $Z$ is not excluded from the model, but it has a different role from $Z_{c}$ in the identification.

The first question is why would the researcher include $Z_{c}$ in the first place. In some cases it may be important to include such controls either because of suspected nonseparable effects or because it is hard to argue the validity of the $I V$ unless it is conditional on $Z_{c}$ as well.

The next question is which variables should be used as $Z$ and which should be used as $Z_{c}$. There is a trade-off: on the one hand, $Z$ can be endogenous, while $Z_{c}$ must be exogenous. On the other hand, $Z$ must be separable from $X$, while $Z_{c}$ may interact with $X$ in arbitrary ways.

Even in an entirely linear model, it is still better to restrict $Z$ only to variables which are believed to be separable from $X$, since the separability of $Z$ is so fundamental to the method. At the same time, notice that $Z$ needs to be only as complex as $X$, and so, for example if $X$ is one continuous variable, it suffices to find just one continuous covariate which can be argued to be separable from $X$. The application Section 6 provides an explicit example of such considerations in an empirical problem.

Remark 3.4 (Non-separability between $X$ and $Z$ ) Although our method cannot identify fully nonparametric non-separable heterogeneous effects in $X$ and $Z$ when $X$ is not binary, i.e. $g$ cannot be a fully nonparametric function of both (see Kasy (2014) for a related partial identification result), we can extend our results to some non-separable models. For example, consider the following examples.

Example 3.4 (Linear model with heterogeneous effects in Z) Consider the varying coefficient model

$$
Y=\beta(Z)^{\prime} X+\varepsilon, \quad \mathbb{E}[\varepsilon \mid Z, T]=\mathbb{E}[\varepsilon \mid Z],
$$

where $X$ is a d-dimensional vector. In this model, we can write

$$
\Delta_{Y}=\beta(Z)^{\prime} \Delta_{X}
$$

which can be used to identify $\beta(Z)$ provided $\Delta_{X} \Delta_{X}^{\prime}$ is non-singular. Under this assumption, we can estimate $\beta(\cdot)$ nonparametrically from local least squares regressions, similar to those carried out for the RRD below. Alternatively, we could specify $\beta(Z)$ as a linear function of $Z$, say $\beta(Z)=\beta_{0}+\beta_{1}^{\prime} Z$, which results in a linear-in-parameters model with endogenous variables $X$ and interactions between $X$ and $Z$, which can be dealt with as in Example 3.3 above. By comparing $\beta(\cdot)$ with the estimator obtained from $\beta=\left(\mathbb{E}\left[\Delta_{X} \Delta_{X}^{\prime}\right]\right)^{-1} \mathbb{E}\left[\Delta_{X} \Delta_{Y}\right]$ we can test for heterogenous marginal effects in $Z$. In the linear specification, this can be done by simply testing if $\beta_{1}=0$. 
Example 3.5 (Nonparametric model with monotonicity) Consider the model

$$
Y=g(X, \varepsilon)
$$

where $\mathbb{E}[\varepsilon \mid X] \neq 0$ with positive probability, and $\varepsilon$ is a structural unobservable error term. Assume $g$ is monotonic in the second argument and denote by $h$ its inverse, i.e. $h(Y, X)=\varepsilon$. Then, by the exclusion restriction $\mathbb{E}[\varepsilon \mid Z, T]=\mathbb{E}[\varepsilon \mid Z]$, it holds

$$
\mathbb{E}[h(Y, X) \mid Z, T=1]-\mathbb{E}[h(Y, X) \mid Z, T=0]=0 \text { a.s. }
$$

Identifying any functional of $h$ from this implicit equation depends on our ability to invert it. Under suitable conditions, including the scale normalization $h(y, \bar{x})=y$ for some known $\bar{x}$ in the support of $X$ (see Matzkin (2003)), we can identify h (up to a constant) from (11).

Remark 3.5 It should be noted that Assumption 2 is different from the completeness between $X$ and $Z$, but it can be understood as a weighted completeness or covariance completeness assumption between these two variables. To see this, define the propensity score $p(z)=\mathbb{E}[T \mid Z=z]$, assume $0<p(z)<1$ a.s., and note that Assumption 2 is equivalent to the implication: $\mathbb{E}[\lambda(X)(T-p(Z)) \mid Z]=0$ a.s. $\Longrightarrow \lambda(X)=\mathbb{E}[\lambda(X)]$ a.s. In contrast, the classical $L_{2}$ completeness between $X$ and $Z$ is equivalent to $\mathbb{E}[\lambda(X) \mid Z]=0$ a.s. $\Longrightarrow \lambda(X)=0$ a.s. (see Newey and Powell (2003)). Furthermore, our identification and estimation can be cast as nonparametric IV with "instrument" $Z$ in the transformed model $Y(T-$ $p(Z))=g(X)(T-p(Z))+\varepsilon(T-p(Z))$. That is, our identifying assumption requires that we can uniquely solve $g$ (up to a constant) from the equation $\operatorname{Cov}(Y, T \mid Z)=\operatorname{Cov}(g(X), T \mid Z)$.

\subsection{Estimation}

In this section we discuss estimation when $g$ is linear in parameters and "first stages" are linear. To see the discussion of the estimation in the nonparametric case, refer to Appendix A.2.1. ${ }^{2}$ As shown in the Appendix, the nonparametric sieve estimator is also linear in parameters, therefore the implementation of this section is also relevant for the nonparametric case.

To better convey ideas, consider first the univariate case and assume $\Delta_{X}$ in (9) is non-zero, so we can write

$$
\beta=\frac{\Delta_{Y}}{\Delta_{X}}
$$

which suggests that we can implement our identification strategy as a Wald estimator by controlling for $Z$ in the structural and first-stage regressions, even though $Z$ and $\varepsilon$ may be correlated, as long as the condition $\mathbb{E}[\varepsilon \mid Z, T]=\mathbb{E}[\varepsilon \mid Z]$ is satisfied.

To formalize this intuition, define $u:=\varepsilon-\mathbb{E}[\varepsilon \mid Z]$, and note that the validity condition can be written as

$$
\mathbb{E}[u \mid Z, T]=0 \text { a.s. }
$$

\footnotetext{
${ }^{2}$ For the related literature on estimation in nonparametric IV see, e.g., Newey and Powell (2003), Hall and Horowitz (2005), Blundell, Chen and Kristensen (2007), Darolles, Fan, Florens and Renault (2011), Horowitz (2011), Chen and Pouzo (2012) and Santos (2012), to mention just a few.
} 
Suppose that $g(X)=\beta^{\prime} X$ and $\mathbb{E}[\varepsilon \mid Z]=\alpha+\gamma^{\prime} Z$, so we can write model (1) as

$$
Y=\alpha+\beta^{\prime} X+\gamma^{\prime} Z+u .
$$

The variable $Z$ is different from a classical "control" variable, since $X$ is still correlated with $u$ (although $u$ is uncorrelated with $Z$ and $T$ by (12)). To deal with the endogeneity of $X$ we can use a TSLS estimator that treats $Z$ as exogenous, as long as our identification conditions are satisfied. Our next result shows this formally when the first stage is given by

$$
\mathbb{E}[X \mid T, Z]=\alpha_{0 X}+\alpha_{1 X} T+\alpha_{2 X}^{\prime} Z+\alpha_{3 X}^{\prime} Z T .
$$

Thus, given a random sample $\left\{\left(Y_{i}, X_{i}, Z_{i}, T_{i}\right)\right\}_{i=1}^{n}$ of $(Y, X, Z, T)$, we propose to estimate $\beta$ with the coefficient of the $X_{i}$ on a TSLS regression of $Y_{i}$ onto $X_{i}$ and $Z_{i}$, using $T_{i}$ and $T_{i} Z_{i}$ as instruments for $X_{i}$, and treating $Z_{i}$ as exogenous. This estimator, denoted by $\widehat{\beta}$, can be implemented with off-the-shelf econometric software. Its asymptotic distribution is well-known, so the novelty of the next result is in the consistency argument.

Theorem 3.2 Let Assumptions 1 and 2 hold with $\mathcal{G}=\left\{b^{\prime} X: b \in \mathbb{R}^{d}\right\}$. Then,

$$
\sqrt{n}(\widehat{\beta}-\beta) \rightarrow_{d} N(0, \Sigma),
$$

where $\Sigma$ is the classical TSLS asymptotic variance (which is assumed to be finite).

Remark 3.6 The arguments above and Monte Carlo simulations in Section 5 suggest that the TSLS will be consistent for $\beta$ even when the conditional means $\mathbb{E}[X \mid T, Z]$ and $\mathbb{E}[\varepsilon \mid Z]$ are non-linear.

Remark 3.7 The choice of $Z$ with regards to identification was discussed in Remark 3.3. However, when choosing $Z$, further consideration should be given to the variance of the estimator which would result from each possible choice. In the parametric setting of this section, the relevance condition is a standard TSLS rank condition that can be tested by traditional methods. Therefore, if there are several variables which satisfy the identification conditions, we recommend that $Z$ be chosen as the variable (or variables) for which $T$ and $T Z$ make up the strongest instruments.

Remark 3.8 We present our method not as an alternative to the classic IV, but as a way to explore separability to identify quantities which cannot be identified with classical IV methods. However, in some instances, even when it is possible to use a classical IV approach, our method may be preferable. For example, in a linear model with a single endogenous variable and a single binary IV, a classical IV approach may be used. However, the IV may be weak, in the sense that $\mathbb{E}[X \mid T=1]-\mathbb{E}[X \mid T=0]$ could be small, but the determinant of $\mathbb{E}\left[\Delta_{X}^{2}\right]$ could still be large. Consider the following example. Suppose that $Z$ is a binary variable which can assume values $\left\{z_{1}, z_{2}\right\}$, with $\mathbb{P}\left(Z=z_{1}\right)=1 / 2$. Suppose that $\mathbb{E}\left[X \mid T=1, Z=z_{1}\right]-\mathbb{E}\left[X \mid T=0, Z=z_{1}\right]=10$, and $\mathbb{E}\left[X \mid T=1, Z=z_{2}\right]-\mathbb{E}\left[X \mid T=0, Z=z_{2}\right]=-10$. In this example, the instrument had a strong, but opposed effect on the subpopulations defined by $Z$. This is an extreme case in which there is no "first-stage" in the classical IV approach, since $\mathbb{E}[X \mid T=1]-\mathbb{E}[X \mid T=0]=0$. However, the strength of our method (in the same units) is given by $\sqrt{\left.\mathbb{E}\left[\Delta_{X}^{2}\right]\right)}=\sqrt{\mathbb{E}\left[\left(10 \cdot 1\left(Z=z_{1}\right)-10 \cdot 1\left(Z=z_{2}\right)\right)^{2}\right]}=10$. 


\section{The Regression Discontinuity Design Case}

\subsection{Identification}

The notation in the Regression Discontinuity standard framework follows from that of the treatment effects literature (Imbens and Angrist (1994)). As such, it is usually concerned with the effects of a binary intervention. However, some examples among the applied works of this literature are interested in the effects of a variable which can take multiple values. ${ }^{3}$ We need to extend the notation to allow for this generalization of the endogenous variable of interest $X$. On that account we will generalize the notation of Hahn, Todd and van der Klaauw (1990) for the constant treatment effects case in the fuzzy design. Our choice is justified because our model cannot allow for nonparametric heterogeneous effects, since the separability of the covariate is fundamental to our approach. Additionally, just as in the fuzzy design, the endogenous variable in our setting may take potentially any value both below and above the threshold, though it must do so with different probabilities.

Let the potential outcome random variable $Y(x)$ satisfy the model ${ }^{4}$

$$
Y(x)=g(x)+\varepsilon .
$$

Let the "predictor" or "running" variable be denoted by $W$, which is univariate and continuously distributed, with threshold $\bar{w}$. The quantities of interest are $\mathbb{E}\left[Y\left(x_{i}\right)-Y\left(x_{j}\right) \mid W=\bar{w}\right]$, for $x_{i} \neq x_{j}$. Given equation (15) and our assumptions below, these are the same as the increments $g\left(x_{i}\right)-g\left(x_{j}\right)$.

As in Section 3, we will use a covariate $Z$ to classify the observations. The validity condition is the same as in the standard RDD setting, after conditioning on $Z$.

Assumption 3 (validity) $\mathbb{E}[\varepsilon \mid W=w, Z]$ is continuous in $w$ at $\bar{w}$ with probability equal to one. ${ }^{5}$

Then, assuming the limits involved are well defined, we obtain

$$
\lim _{w \downarrow \bar{w}} \mathbb{E}[Y \mid W=w, Z]-\lim _{w \uparrow \bar{w}} \mathbb{E}[Y \mid W=w, Z]=\lim _{w \downarrow \bar{w}} \mathbb{E}[g(X) \mid W=w, Z]-\lim _{w \uparrow \bar{w}} \mathbb{E}[g(X) \mid W=w, Z] .
$$

The right hand side defines implicitly a linear operator of $g$. We denote it by $A g$, with some abuse of notation. Assumption 2 can be interpreted in the context of the RDD analogously to the cases discussed in Section 3.1. Therfore, if Assumptions 2 and 3 hold, $g$ is identified up to a constant (see Theorem 3.1 for the proof). The fundamental requirement is that the difference between the distribution of $X$ conditional on $W$ and $Z$ at the limits from above and below $\bar{w}$ vary sufficiently with $Z$. The following example shows the translation of this condition for the multivariate linear case.

Example 4.1 Consider the linear model (where $X$ may be multivariate) under Assumption 3,

$$
Y=\alpha+\beta^{\prime} X+\varepsilon,
$$

\footnotetext{
${ }^{3}$ See Chay and Greenstone (2005), Carpenter and Dobkin (2009) and Brollo, Nannicini, Perotti and Tabellin (2013), among many others, for empirical examples with continuous endogenous variables in the RDD.

${ }^{4}$ Hahn, Todd and van der Klaauw (1990) denote the potential outcome $y_{i}=\alpha_{i}+x_{i} \cdot \beta$. In our case, $\alpha$ is represented by $\varepsilon$ and $g(x)$ is the generalization of $x \cdot \beta$.

${ }^{5}$ Assumption (A1) in Hahn, Todd and van der Klaauw (1990).
} 
so that

$$
\lim _{w \downarrow \bar{w}} \mathbb{E}[Y \mid W=w, Z]-\lim _{w \uparrow \bar{w}} \mathbb{E}[Y \mid W=w, Z]=\beta^{\prime}\left(\lim _{w \downarrow \bar{w}} \mathbb{E}[X \mid W=w, Z]-\lim _{w \uparrow \bar{w}} \mathbb{E}[X \mid W=w, Z]\right),
$$

or using the short notation $\delta_{Y}=\beta^{\prime} \delta_{X}$, where we denote for a generic random vector $V$

$$
\delta_{V}=\lim _{w \downarrow \bar{w}} \mathbb{E}[V \mid W=w, Z]-\lim _{w \uparrow \bar{w}} \mathbb{E}[V \mid W=w, Z],
$$

assuming the limits exist. Assumption 2 in this context is equivalent to $\mathbb{E}\left[\delta_{X} \delta_{X}^{\prime}\right]$ being positive definite. In other words, we need that the variation across the threshold in the mean of $X$ for a given $Z$ vary in a linearly independent way.

\subsection{Estimation}

In this section we discuss estimation for the RDD case when $g$ is linear, as in Example 4.1. To see the discussion of the estimation in the nonparametric case, refer to Appendix A.2.2. We write

$$
Y=\alpha+\beta^{\prime} X+\gamma^{\prime} Z+u
$$

where $\mathbb{E}[u \mid W=w, Z]$ is continuous in $w$ at $\bar{w}$ with probability one, and satisfies further conditions below (see Assumption 4 in the Appendix). Without loss of generality we take hereinafter $\bar{w}=0$.

We assume that the first stage is given by the semiparametric varying coefficient models

$$
\mathbb{E}[X \mid Z, W]=: \alpha_{0 X}(W)+\alpha_{1 X}^{\prime}(W) Z,
$$

where $\alpha_{0 X}(\cdot)$ and $\alpha_{1 X}(\cdot)$ are unknown functions of $W$. This specification overcomes the "curse of dimensionality" problem when the dimension of $Z$ is large, as can be the case in applications.

Model (18) provides a natural extension of (14) to the continuous case. In fact, if we approximate $\alpha_{0 X}(\cdot)$ and $\alpha_{1 X}(\cdot)$ locally at $W=0$ by constants to each side of the threshold, the resulting model is (14), where the IV is $T_{i}=1\left(W_{i} \geq 0\right)$. This suggests that a local version of the proposed TSLS estimator in the IV case (see Section 3.2)) will provide valid inference on $\beta$.

However, it is well known that the local constant kernel estimator has generally worse bias properties than the local linear kernel estimator at discontinuity points; see Fan and Gijbels (1996). For this reason, we suggest implementing our identification strategy for the RDD with a local linear estimator instead. This corresponds to the use of linear (as opposed to constants) approximations for $\alpha_{0 X}(\cdot)$ and $\alpha_{1 X}(\cdot)$ around each side of the threshold $\bar{w}=0$. More generally, we can apply higher order approximations, resulting in local polynomial estimators of a certain degree. All these estimators can be implemented as TSLS estimators by defining a suitable vector of instruments.

Specifically, consider the uniform kernel

$$
k_{h i}=1\left(-h \leq W_{i} \leq h\right),
$$

where $h$ is a bandwidth parameter satisfying that $h \rightarrow 0$ as $n \rightarrow \infty$ and other conditions below. Then, our estimator for RDD can be easily implemented by restricting the sample $\left\{\left(Y_{i}, X_{i}, Z_{i}, W_{i}\right)\right\}_{i=1}^{n}$ to 
only those observations such that $-h \leq W_{i} \leq h$, and running a TSLS regression of $Y$ onto $X$ and $Z$, treating $Z_{i}$ as exogenous, and using

$$
V_{i}=\left(T_{i}, Z_{i} \times T_{i}, W_{i}, T_{i} \times W_{i}, Z_{i} \times W_{i}, Z_{i} \times T_{i} \times W_{i}\right)^{\prime}
$$

as a vector of "instruments" for $X$. Denote by $\tilde{\beta}$ the corresponding TSLS estimator of the coefficient of $X$. In Section A.1.2 in the Appendix we prove the asymptotic normality of a slightly generalized version of $\tilde{\beta}$ where a general kernel, not necessarily the uniform kernel (19), is used. For simplicity, we only consider a univariate $X$ and $Z$. The multivariate case can be proved analogously.

Theorem 4.1 Let Assumption 2 hold with $\mathcal{G}=\left\{b^{\prime} X: b \in \mathbb{R}^{d}\right\}$, as well as Assumption 3 and Assumption 5 in Section A.1.2 in the Appendix. Then

$$
\sqrt{n h}(\tilde{\beta}-\beta) \rightarrow_{d} N\left(0, Q_{2} \Omega Q_{2}^{\prime}\right)
$$

where expressions for $Q_{2}$ and $\Omega$ are given in Section A.1.2.

The asymptotic variance of $\tilde{\beta}$ can be consistently estimated by the standard TSLS asymptotic variance. Implementation of $\tilde{\beta}$ requires the choice of a bandwidth parameter $h$. We can choose $h$ along the lines suggested in Calonico, Cattaneo and Titiunik (2014), which can be adapted to the presence of covariates.

Note that the way in which we introduce covariates in our RDD approach is different from how it is traditionally done, which does not account for interaction terms between the covariates and the running variable; see, e.g., Imbens and Lemieux (2008). Our arguments suggest that, provided the separability conditions that we impose are satisfied, including covariates in this way may allow us to identify quantities which would otherwise not be identifiable with the current state-of-the-art in RDD literature.

\section{Monte Carlo Simulations}

\subsection{The case with a binary IV}

Consider the following data generating process (DGP):

$$
\begin{aligned}
Y & =\alpha+\beta_{1} X_{1}+\beta_{2} X_{2}+\gamma_{z} Z+u, \\
Z & =\alpha_{z} u+\varepsilon_{z}, \\
X_{1} & =\alpha_{01}+\alpha_{11} T+\alpha_{21} Z+\alpha_{31} T \times Z+\varepsilon_{1}, \\
X_{2} & =\alpha_{02}+\alpha_{12} T+\alpha_{22} Z+\alpha_{32} T \times Z+\varepsilon_{2},
\end{aligned}
$$

where $\left(u, \varepsilon_{z}, \varepsilon_{1}, \varepsilon_{2}\right)$ are independent standard normal random variables independent of $T$, which is distributed as Bernoulli random variable with probability $p=0.5$. This is a linear model with three endogenous variables and one binary instrument. The classical order condition of standard IV does not hold in this case, and hence, classical IV is unable to identify the marginal effects $\beta_{1}$ and $\beta_{2}$. For 
identification of the marginal effects, our method requires that Assumption 2 holds, which in this case is equivalent to

$$
\operatorname{det}\left|\begin{array}{ll}
\alpha_{11} & \alpha_{31} \\
\alpha_{12} & \alpha_{32}
\end{array}\right| \neq 0 .
$$

The parameters in the structural equation are set at $\alpha=0, \beta_{1}=1, \beta_{2}=2$ and $\gamma_{z}=1$. We set $\alpha_{01}=\alpha_{02}=\alpha_{21}=\alpha_{22}=0$, and $\alpha_{z}=1$, so $Z$ is endogenous. Table 2 provides the average bias and Mean Squared Error (MSE) based on 10,000 Monte Carlo simulations, sample sizes $n=100,300,500$ and 1000, and several values for $\left(\alpha_{11}, \alpha_{31}, \alpha_{12}, \alpha_{32}\right)$. There are three variables in the structural equation and three in each reduced form equation, and therefore, the TSLS estimator is an IV estimator that treats $T$ and the interaction term $T \times Z$ as instruments.

We observe a satisfactory bias performance uniformly over all parameter values. For the first two cases $\left(\alpha_{11}=1, \alpha_{31}=0, \alpha_{12}=0, \alpha_{32}=1\right)$ and $\left(\alpha_{11}=0, \alpha_{31}=1, \alpha_{12}=1, \alpha_{32}=0\right)$ the sample variance of the estimators is already small for small sample sizes as 100, and it decreases to zero with the sample size, in accordance with the consistency of the estimator. For the parameter values $\left(\alpha_{11}=1.25, \alpha_{31}=1, \alpha_{12}=1, \alpha_{32}=1.25\right)$ identification is much weaker, and larger sample sizes are required for a good performance, as expected. Overall, these results provide supporting evidence of the robustness of our identification strategy to the endogeneity of $Z$.

Table 2: IV Case

\begin{tabular}{|c|c|c|c|c|c|c|c|c|}
\hline$\alpha_{11}$ & $\alpha_{31}$ & $\alpha_{12}$ & $\alpha_{32}$ & $n$ & $\operatorname{Bias}\left(\beta_{1}\right)$ & $M S E\left(\beta_{1}\right)$ & $\operatorname{Bias}\left(\beta_{2}\right)$ & $M S E\left(\beta_{2}\right)$ \\
\hline \multirow{4}{*}{1} & \multirow{4}{*}{0} & \multirow{4}{*}{0} & \multirow{4}{*}{1} & 100 & -0.0041 & 0.0252 & 0.0013 & 0.0123 \\
\hline & & & & 300 & 0.0015 & 0.0070 & 0.0000 & 0.0034 \\
\hline & & & & 500 & -0.0014 & 0.0041 & 0.0000 & 0.0020 \\
\hline & & & & 1000 & 0.0001 & 0.0020 & 0.0003 & 0.0010 \\
\hline \multirow{4}{*}{0} & \multirow{4}{*}{1} & \multirow{4}{*}{1} & \multirow{4}{*}{0} & 100 & -0.0002 & 0.0121 & 0.0009 & 0.0243 \\
\hline & & & & 300 & -0.0004 & 0.0035 & 0.0002 & 0.0070 \\
\hline & & & & 500 & 0.0002 & 0.0020 & 0.0000 & 0.0041 \\
\hline & & & & 1000 & -0.0001 & 0.0010 & 0.0004 & 0.0020 \\
\hline \multirow{4}{*}{1.25} & \multirow{4}{*}{1} & \multirow{4}{*}{1} & \multirow{4}{*}{1.25} & 100 & 0.1247 & 259.7900 & -0.0897 & 193.4900 \\
\hline & & & & 300 & 0.0528 & 11.1740 & -0.0434 & 7.2709 \\
\hline & & & & 500 & -0.0065 & 0.2693 & 0.0053 & 0.2085 \\
\hline & & & & 1000 & 0.0007 & 0.0157 & -0.0003 & 0.0136 \\
\hline
\end{tabular}

10000 Monte Carlo Simulations.

In the second set of simulations we provide finite-sample evidence of the robustness of the estimator 
to the failure of the linear specifications of the first stages. Consider now the DGP:

$$
\begin{aligned}
& Y=\alpha+\beta_{1} D+\beta_{2} D 1(D>0)+\gamma_{z} Z+u, \\
& Z=\alpha_{z} u+\varepsilon_{z} \\
& D=\alpha_{d} Z+\gamma_{d} T \times Z+\varepsilon_{d},
\end{aligned}
$$

where $\left(u, \varepsilon_{z}, \varepsilon_{d}\right)$ are independent standard normal random variables, independent of $T$, which is again distributed as Bernoulli with probability $p=0.5$. This corresponds to a linear model

$$
Y=\alpha+\beta_{0}^{\prime} X+\varepsilon
$$

where $\beta_{0}=\left(\beta_{1}, \beta_{2}\right)^{\prime}, X=(D, D 1(D>0))^{\prime}$ and $\varepsilon=\gamma_{z} Z+u$. Here

$$
\mathbb{E}[D \mid Z, T=1]-\mathbb{E}[D \mid Z, T=0]=\gamma_{d} Z,
$$

so $\gamma_{d}$ controls the identification strength. Note that when $\alpha_{z} \neq 0 Z$ is endogenous. Since there is only one binary IV, $T$, standard IV methods cannot be applied in this example. Note also that under this DGP the difference of conditional means $\Delta_{X}$ is nonlinear in $Z$ in its second component. However, it can be shown that our estimator based on the linearity assumption is still consistent, as we illustrate with some Monte Carlo experiments. This shows the robustness of our estimator to the failure of the linearity assumption in the conditional mean $\mathbb{E}[X \mid Z, T]=\alpha_{0 X}(T)+\alpha_{1 X}^{\prime}(T) Z$.

Table 3 provides the average bias and MSE based on 10000 Monte Carlo simulations. In all cases $\alpha=0, \gamma_{z}=1, \beta_{1}=1, \beta_{2}=2, \alpha_{z}=1, \alpha_{d}=1$. We consider two levels of identification, "moderate" $\gamma_{d}=1$ and "high" $\gamma_{d}=2$.

Table 3: IV Case - Misspecified Model

\begin{tabular}{rrrrrr}
\hline \hline$\gamma_{d}$ & $n$ & $\operatorname{Bias}\left(\beta_{1}\right)$ & $M S E\left(\beta_{1}\right)$ & $\operatorname{Bias}\left(\beta_{2}\right)$ & $M S E\left(\beta_{2}\right)$ \\
\hline \hline & 100 & 0.00107 & 0.48403 & -0.01446 & 2.64655 \\
1 & 300 & -0.00067 & 0.01124 & 0.00039 & 0.03006 \\
& 500 & 0.00001 & 0.00638 & 0.00042 & 0.01664 \\
& 1000 & 0.00003 & 0.00303 & -0.00072 & 0.00802 \\
\hline \hline & 100 & -0.00160 & 0.00924 & 0.00320 & 0.02321 \\
2 & 300 & -0.00065 & 0.00252 & 0.00089 & 0.00645 \\
& 500 & 0.00000 & 0.00148 & 0.00024 & 0.00385 \\
& 1000 & 0.00031 & 0.00074 & -0.00019 & 0.00189 \\
\hline \hline
\end{tabular}

10000 Monte Carlo Simulations.

The reported results show that the estimator is still consistent, despite the misspecification of the first stages. Estimates of $\beta_{2}$ require larger sample sizes than those of $\beta_{1}$ to achieve the same level of 
precision and bias performance. There is an efficiency loss in estimating $\beta_{2}$ relative to $\beta_{1}$, probably due to the nonlinearities in $\mathbb{E}[D 1(D>0) \mid Z, T=j]$ for $j=0,1$. As expected, the results improve with the identification strength. In sum, these simulations provide finite sample evidence of a satisfactory performance of the TSLS estimator and its robustness to the endogeneity of the covariates and the nonlinearity of the first stages.

\subsection{The RDD case}

We consider now a third DGP to evaluate the new RDD estimator. The DGP is given by:

$$
\begin{aligned}
& Y=\alpha+\beta X+\gamma Z+u \\
& T=1(W \geq 0) \\
& X=\alpha_{0}+\alpha_{1} T+\alpha_{2} Z+\alpha_{3} T \times Z+\varepsilon_{X}
\end{aligned}
$$

where $\left(u, \varepsilon_{X}, W, Z\right)$ are independent and identically distributed as standard normals. Note that with this design

$$
\lim _{w \downarrow 0} \mathbb{E}[X \mid W=w, Z]=\alpha_{0}+\alpha_{1}+\left(\alpha_{2}+\alpha_{3}\right) Z
$$

and

$$
\lim _{w \uparrow 0} \mathbb{E}[X \mid W=w, Z]=\alpha_{0}+\alpha_{2} Z .
$$

Therefore, $\delta_{X i}=\alpha_{1}+\alpha_{3} Z_{i}$. On the other hand, since $\mathbb{E}[Z \mid W]=0$,

$$
\lim _{w \downarrow 0} \mathbb{E}[X \mid W=w]=\alpha_{0}+\alpha_{1}
$$

and

$$
\lim _{w \uparrow 0} \mathbb{E}[X \mid W=w]=\alpha_{0} .
$$

Therefore, in this model $\alpha_{1}$ controls the level of identification of the classical RDD, whereas $\alpha_{1}$ and $\alpha_{3}$ control that for the new RDD estimator. We compare the classical RDD estimator $\left(\widehat{\beta}_{R D D}\right)$ and the new RDD estimator $(\tilde{\beta})$. We consider the parameter values $(\alpha, \beta, \gamma)=(0,1,0),\left(\alpha_{0}, \alpha_{2}, \alpha_{3}\right)=(0,1,1)$ and several values of $\alpha_{1}$. We implement both estimators as in Section 4.2 with a uniform kernel (19) and a bandwidth $h=2 n^{-1 / 4}$. In particular, the classical RDD estimator $\widehat{\beta}_{R D D}$ is obtained as a TSLS estimator in the restricted sample $\left\{\left(Y_{i}, X_{i}, Z_{i}, W_{i}\right):-h \leq W_{i} \leq h\right\}_{i=1}^{n}$, where the structural equation is given by

$$
Y_{i}=\alpha+\beta X+\gamma Z+u
$$

and the first stage is given by

$$
\mathbb{E}[X \mid Z, W]=\alpha_{0 X}+\alpha_{1 X} T+\alpha_{2 X} Z+\alpha_{4 X} W+\alpha_{5 X} T W
$$

whereas $\tilde{\beta}$ uses the first stage

$$
\mathbb{E}[X \mid Z, W]=\alpha_{0 X}+\alpha_{1 X}^{\prime} V+\alpha_{2 X} Z
$$


where $V$ is defined in (20).

Table 4 reports the average bias and MSE based on 10000 Monte Carlo simulations. For $\alpha_{1}=0$, the classical RDD is not able to identify the parameter $\beta$, and, as expected, has a MSE that does not converge to zero for large $n$. In contrast, our estimator $\tilde{\beta}$ presents a satisfactory performance, with small MSEs that decrease with the sample size. The classic RDD requires a value of $\alpha_{1}=2$ to achieve comparable results to those of the new RDD. Unreported results with other bandwidths and with other parameter values for $\left(\alpha_{0}, \alpha_{2}, \alpha_{3}\right)$ show a similar behavior.

Table 4: Average Bias and MSE: RDD

\begin{tabular}{rrcccc}
\hline \hline$\alpha_{1}$ & $n$ & \multicolumn{2}{c}{ Bias } & \multicolumn{2}{c}{$M S E$} \\
\hline & & $\widehat{\beta}_{R D D}$ & $\tilde{\beta}$ & $\widehat{\beta}_{R D D}$ & $\tilde{\beta}$ \\
\hline \hline & 100 & -0.0048 & 0.0016 & 0.8018 & 0.0650 \\
& 300 & 0.0018 & -0.0004 & 0.8084 & 0.0275 \\
& 500 & 0.0024 & -0.0014 & 0.8077 & 0.0182 \\
& 1000 & -0.0014 & 0.0016 & 0.6842 & 0.0107 \\
\hline \hline & 100 & 0.0002 & 0.0006 & 0.0784 & 0.0348 \\
& 300 & -0.0008 & -0.0006 & 0.0292 & 0.0139 \\
1 & 500 & -0.0018 & -0.0016 & 0.0192 & 0.0094 \\
& 1000 & 0.0016 & 0.0015 & 0.0104 & 0.0053 \\
\hline \hline & 100 & 0.0005 & 0.0007 & 0.0181 & 0.0141 \\
& 300 & -0.0004 & -0.0004 & 0.0071 & 0.0056 \\
2 & 500 & -0.0008 & -0.0010 & 0.0047 & 0.0038 \\
& 1000 & 0.0007 & 0.0009 & 0.0026 & 0.0021 \\
\hline \hline
\end{tabular}

10000 Monte Carlo Simulations.

\section{An Application to the Estimation of the Effects of Air Pollution on House Prices}

Our identification strategy is applied in this section to the problem of estimating the effects of pollution on house prices, as in Chay and Greenstone (2005). The concern with endogeneity in this problem is warranted, since counties may differ from each other in many ways which may not be predicted by their observable characteristics and amenities. Chay and Greenstone base their identification strategy on an instrumental variable approach, which takes advantage of the quasi-experiment generated by the Clean Air Act around the time it was first implemented. This application is particularly illustrative 
of our approach because of the nonattainment sharp threshold, and thus it is also possible to pursue identification in an RDD framework.

As explained in Remark 3.3, it is not necessary that all the covariates be separable from $X$ in the model. Let $Y$ denote the change between 1970 and 1980 in the logs of the county's median property values, $X$ is the change between 1970 and 1980 in the geometric mean total suspended particulates (TSP) across all monitors in the county, $T$ is the county's attainment status in 1975 according to the Clean Air Act, and $Z_{c}$ and $Z$ denote vectors of further variables which are used by Chay and Greenstone as controls in their model specification (2) (Chay and Greenstone (2005), p. 411). We can estimate a model

$$
Y=g\left(X, Z_{c}\right)+\varepsilon
$$

with the exclusion restriction $\mathbb{E}\left[\varepsilon \mid T, Z_{c}, Z\right]=\mathbb{E}[\varepsilon \mid Z]$, thus allowing a set of covariates $Z_{c}$ to be nonseparable from $X$. We can generalize Chay and Greenstone (2005)'s approach in two directions. First, even though the instrument $T$ is binary we are able to identify $g$ when it is more general than simply linear. Second, since $Z$ in our approach may be endogenous, the covariates which we choose to use as $Z$ need no longer be assumed exogenous.

In choosing which covariates are part of $Z$, we must be concerned with its separability from $X$. The issue is that counties' taste for pollution may differ as a function of some of the covariates. Because of this, it is unadvisable to use covariates such as, for example, the percent change in income, education levels, racial composition and unemployment in the county population, as these could be reasonably assumed to influence the population's taste in pollution. The covariates which we believe are most likely to be separable are the county's changes between 1970 and 1980 in the percent spending in highways, health and education. We considered estimators of our method with each of those variables separately and also together, as can be seen in Table 5 .

The estimation is done using the same data set as in Chay and Greenstone (2005) as well as the exact same covariate specification. The first column in Table 4 shows the results of a standard IV estimation of the effects of pollution change, which is what is done in Chay and Greenstone (2005). The replicated results are, not surprisingly, identical to that paper. Columns A to D show the results of our estimation approach using different variables as the separable classification covariate. Row I uses a specification without exogenous control variables. Although in specification I our results are of similar magnitude to the standard IV, they are smaller and vary depending on the covariate. They are particularly smaller when all three covariates are used. We believe that this happens because our estimator operates under the assumption that the IV is valid only conditional on the separable covariate $(\mathbb{E}[\varepsilon \mid T, Z]=\mathbb{E}[\varepsilon \mid Z])$. Indeed, Chay and Greenstone do not suppose that their IV is valid, but only that it is valid conditional on controls. Row II shows the results conditional on controls. There the identifying assumption of the classic IV approach is that $\mathbb{E}\left[\varepsilon \mid T, Z_{c}, Z\right]=0$, while our identifying assumption is that $\mathbb{E}\left[\varepsilon \mid T, Z_{c}, Z\right]=\mathbb{E}[\varepsilon \mid Z]$. Nevertheless, our results generally confirm the estimates found by Chay and Greenstone.

Additionally, we ran the same regressions with the other covariates used in the model. Although we did not feel secure to include them as separable, most of them confirm our findings very closely. In 


\section{Table 5: Estimation Results - Linear Case, IV Approach}

\begin{tabular}{cccccc}
\hline \hline & $\mathrm{IV}$ & $\mathrm{A}$ & $\mathrm{B}$ & $\mathrm{C}$ & $\mathrm{D}$ \\
\hline \hline $\mathrm{I}$ & -.347 & -.340 & -.327 & -.317 & -.327 \\
& $(.140)$ & $(.138)$ & $(.136)$ & $(.134)$ & $(.135)$ \\
\hline \multirow{2}{*}{$\mathrm{II}$} & -.203 & -.208 & -.202 & -.203 & -.208 \\
& $(.093)$ & $(.094)$ & $(.093)$ & $(.093)$ & $(.093)$ \\
\hline \hline
\end{tabular}

Table 5: Columns A to D use our approach with $Z$ equal to the change from 1970 to 1980 in the \% spending on highways (A), health (B), and education (C). In column (D), $Z$ is the vector of all three variables. Specification I has no exogenous covariates $Z_{c}$. Specification II uses as exogenous covariates $Z_{c}$ the the exact same specification as in Chay and Greenstone (2005).

fact, after controlling for covariates, only 5 of our regressions yielded results far from -.203: the number of houses built between 1970 and 1980, the rate of vacancies in 1980, change in income per-capita, the change in government revenue per-capita and the change in the fraction of the population with at least a college degree. However, we should point out that even for these covariates, the differences between our estimates and -.203 was still less than .1.

The advantages of our method are better showcased in the nonlinear case, where the classical instrumental variables methods cannot identify marginal effects with a single instrumental variable. We estimate a partially linear model $Y=g(X)+Z_{c}^{\prime} \gamma+\varepsilon$, assuming that $\mathbb{E}\left[\varepsilon \mid T, Z_{c}, Z\right]=\mathbb{E}[\varepsilon \mid Z]$, where $g$ is a connected piecewise linear function and $Z_{c}$ is the entire specification of controls in Chay and Greenstone (2005) except for the variables in $Z$. Figure 1 has three pieces, which connect at the terciles of the distribution of $X$. Hence, we can write $g(x)=a_{1} \psi_{1}^{3}(x)+a_{2} \psi_{2}^{3}(x)+a_{3} \psi_{3}^{3}(x)$, where the $\psi_{j}^{3}(x)$ are the elements of a B-spline basis of degree 1 and smoothness 0 with knots at the terciles just described. In practice this is the same as if we had three endogenous variables $\psi_{1}^{3}(X), \psi_{2}^{3}(X)$ and $\psi_{3}^{3}(X)$. For $Z$ we used the three variables in column D in Table 5 (call them High, Health and $E d u c)$ expanded into the elements of the B-spline basis. So, with some abuse of notation $Z=$ $\left(\psi_{1}^{3}(\text { High }), \psi_{2}^{3}(\text { High }), \psi_{3}^{3}(H i g h), \psi_{1}^{3}(\text { Health }), \psi_{2}^{3}(\text { Health }), \psi_{3}^{3}(\text { Health }), \psi_{1}^{3}(\text { Educ }), \psi_{2}^{3}(\text { Educ }), \psi_{3}^{3}(\text { Educ })\right)^{\prime}$. Figures 2 to 4 are obtained analogously.

Our results qualitatively confirm the findings of the linear case, but it is important to notice that the effect may be even more negative than predicted in the linear case in the majority of the domain. Also, interestingly, for a large part of the domain the effect seems to go in the opposite direction. In fact, for small reductions in pollution, the derivatives are not only positive, but rather high.

Figures 1 to 4 are representative of the patterns we found when we tried other strategies. For example, we also used as $Z$ each of the elements used in columns A to C in Table 5 separately (i.e. for $g$ with three pieces we $\operatorname{did} Z=\left(\psi_{1}^{3}(H i g h), \psi_{2}^{3}(H i g h), \psi_{3}^{3}(H i g h)\right)^{\prime}$, and we also expanded the elements of $Z$ in other ways, for example into two piece B-splines $\left(Z=\left(\psi_{1}^{2}(H i g h), \psi_{2}^{2}(H i g h), \psi_{1}^{2}(\right.\right.$ Health $), \psi_{2}^{2}($ Health $)$, 
Figure 1: 3 pieces

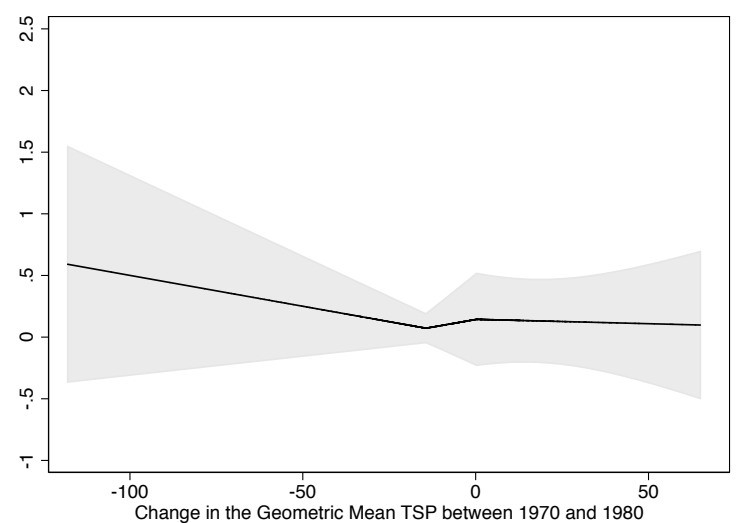

Figure 3: 5 pieces

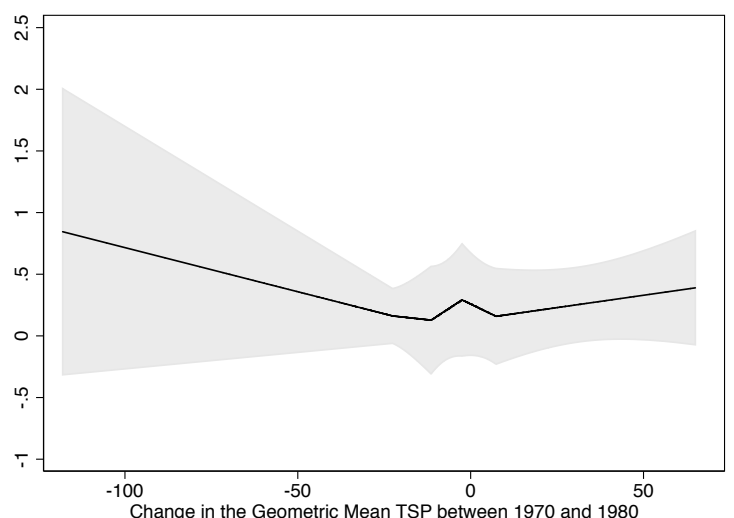

Figure 2: 4 pieces

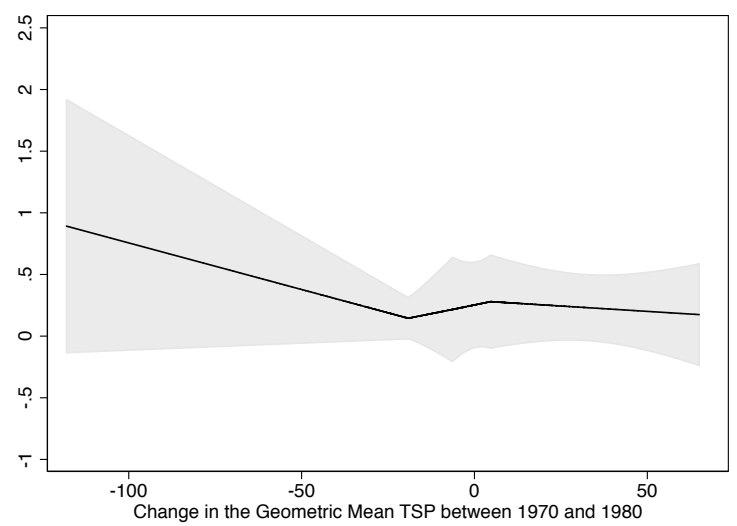

Figure 4: 6 pieces

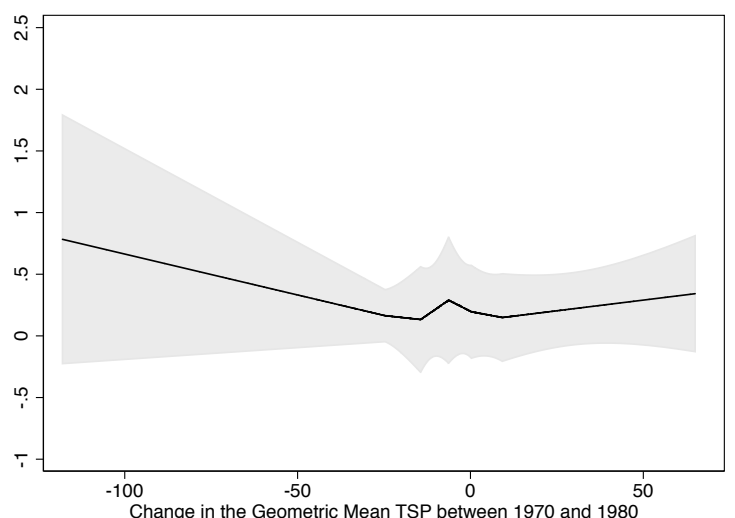

Figures 1 to 4: Nonlinear case - IV approach. Curves are the results of our approach with exogenous covariates, and $Z$ as in column $\mathrm{D}$ in Table 5. The domain of each piece is determined by the quantiles. For instance, Figure 2's pieces connect at the 25th, 50t and 75th quantiles of the change in the geometric Mean TSP between 1970 and 1980 .

$\left.\left.\psi_{1}^{2}(E d u c), \psi_{2}^{2}(E d u c)\right)^{\prime}\right)$, four piece B-splines, etc., all with very similar results. The standard errors get substantially larger as we increase the number of pieces in $g$, but they are not affected (and seem in fact to decrease) as we increase the number of elements in $Z$.

For the RDD approach, Chay and Greenstone (2005) report the results with bandwidth $h=25$ and local quadratic estimators in the paper, which can be seen in the fourth row of results in Table 6 for direct comparisons. The results show some variability and dependence on the bandwidth size, but qualitatively they confirm the findings of the standard IV case. For the smallest bandwidth $h=15$ our results are considerably smaller than the standard RDD, and they show large variations depending on the chosen $Z$. However, for the larger bandwidths they become more stable and similar to the standard RDD, although still smaller (and generally closer to the numbers found in the IV case).

We can also apply our method for the RDD to identify the response curve in the nonlinear case, which cannot be achieved with the standard RDD identification method. Figures 5 to 8 below are very similar to the curves estimated in the IV case, and they still show the curious pattern of positive 


\section{Table 6: Estimation Results - Linear Case, RDD Approach}

\begin{tabular}{|c|c|c|c|c|c|c|}
\hline $\mathrm{h}$ & $\mathrm{p}$ & RDD & A & B & $\mathrm{C}$ & $\mathrm{D}$ \\
\hline \multirow{4}{*}{15} & \multirow{2}{*}{1} & -.305 & -.155 & -.145 & -.173 & -.074 \\
\hline & & $(.246)$ & $(.206)$ & $(.171)$ & $(.175)$ & $(.148)$ \\
\hline & \multirow{2}{*}{2} & -.292 & -.019 & -.120 & -.137 & .034 \\
\hline & & $(.240)$ & $(.179)$ & $(.157)$ & $(.163)$ & $(.130)$ \\
\hline \multirow{4}{*}{25} & \multirow{2}{*}{1} & -.249 & -.233 & -.192 & -.234 & -.182 \\
\hline & & $(.142)$ & $(.137)$ & $(.122)$ & $(.131)$ & $(.117)$ \\
\hline & \multirow{2}{*}{2} & -.275 & -.169 & -.212 & -.253 & -.112 \\
\hline & & (.141) & $(.119)$ & $(.120)$ & $(.132)$ & $(.106)$ \\
\hline \multirow{4}{*}{35} & \multirow{2}{*}{1} & -.374 & -.305 & -.307 & -.332 & -.227 \\
\hline & & $(.156)$ & $(.138)$ & $(.132)$ & $(.145)$ & $(.118)$ \\
\hline & \multirow{2}{*}{2} & -.330 & -.208 & -.267 & -.289 & -.151 \\
\hline & & $(.142)$ & $(.114)$ & $(.121)$ & $(.131)$ & (.101) \\
\hline \multirow{4}{*}{45} & 1 & -.419 & -.405 & -.364 & -.379 & -.311 \\
\hline & 1 & $(.139)$ & $(.134)$ & $(.124)$ & $(.131)$ & $(.115)$ \\
\hline & \multirow{2}{*}{2} & -.391 & -.373 & -.342 & -.355 & -.310 \\
\hline & & $(.136)$ & $(.128)$ & $(.119)$ & $(.126)$ & $(.115)$ \\
\hline
\end{tabular}

Table 6: Columns A to D use our approach with $Z$ equal to the change from 1970 to 1980 in the $\%$ spending on highways (A), health (B), and education (C). In column (D), $Z$ is the vector of all three variables. $h$ is the bandwidth and $p$ is the degree of the local polynomial. We used local linear and local quadratic estimators because Chay and Greenstone (2005) reported estimates use the local quadratic.

derivatives for part of the support. However, the slopes are substantially smaller in the entire domain. The standard errors are also smaller, which is in part due to the fact that there are no covariates.

These curves are representative of the patterns we find when we try other strategies. For example, Figures 5 to 8 use the local quadratic instead of the local linear estimator because we wanted to compare our results to Chay and Greenstone (2005)'s which use the local quadratic estimator in their approach. However, the results with the local linear are nearly identical. We also experimented using different combinations of variables to form $Z$, just as described in the standard IV case (see p. 23 after Figures 1 to 4$)$. The results are again very similar, with one interesting difference: when we used the $\%$ spending on education (column C) the curve slopes downward for the highest quantiles. 
Figure 5: 3 pieces

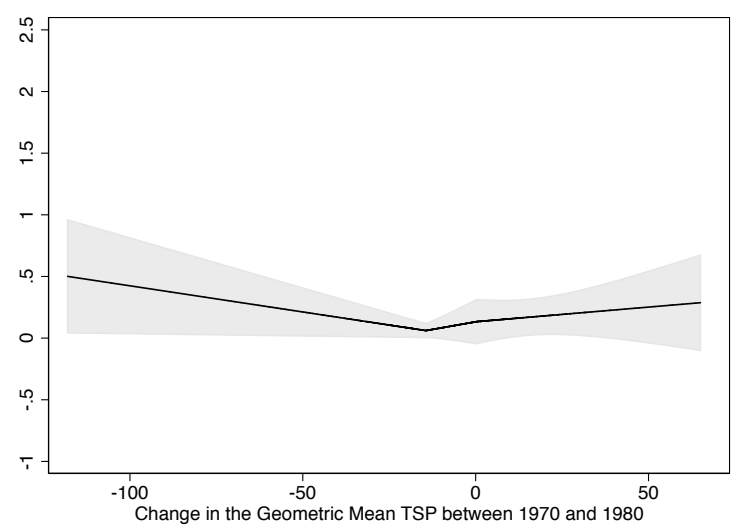

Figure 7: 5 pieces

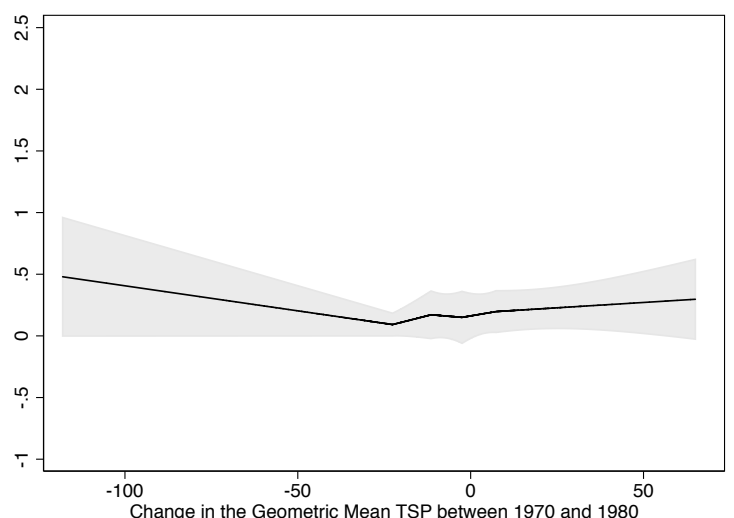

Figure 6: 4 pieces

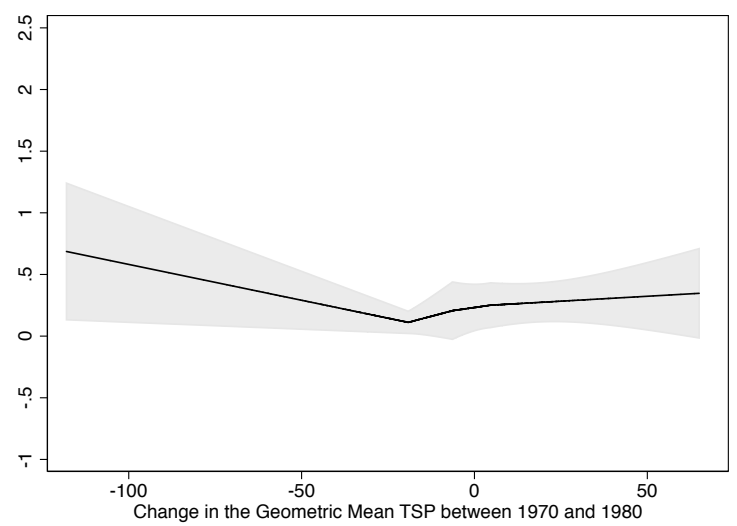

Figure 8: 6 pieces

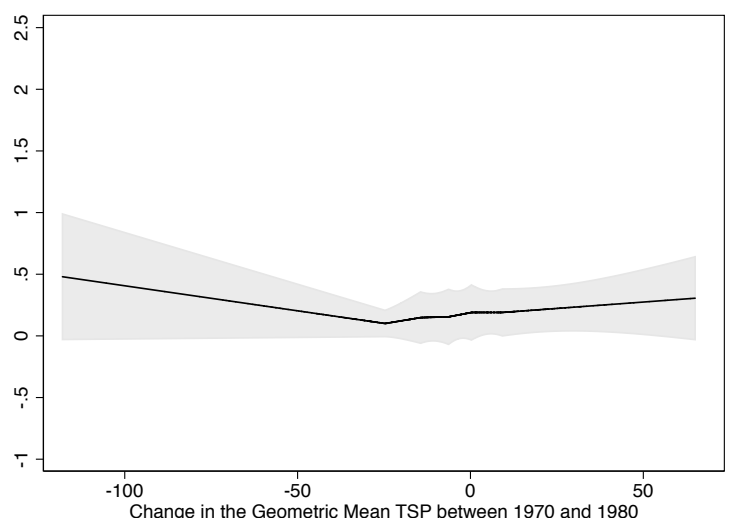

Figures 5 to 8: Nonlinear case - RDD approach. Curves are the results of our approach using $Z$ as in column D in Table 5 and the local quadratic (bandwidth is $h=25$ ). The domain of each piece is determined by the quantiles. For instance, Figure 6's pieces connect at the 25th, 50t and 75th quantiles of the change in the geometric Mean TSP between 1970 and 1980

\section{Conclusions}

In this paper we have proposed a strategy to identify marginal effects of "complex" variables using a binary IV in the presence of other possible endogenous covariates. The strategy hinges on the heterogeneity of the "first stages" in those covariates, and it can be extended to the RDD. It can be applied to parametric, semiparametric and nonparametric settings. In models that are linear in parameters (which also include nonparamatric models estimated by sieves), the identification strategy can be implemented with a simple TSLS estimator that treats the covariates as if they were exogenous, and runs a first stage with interactions between the Binary IV and the covariates. Thus, our identification strategy can be implemented with off-the-shelf econometric software. Monte Carlo simulations show that this TSLS estimator performs well in practice, and it is robust to endogeneity of covariates and misspecification of the linear conditional expectations.

In the RDD case, we propose a semiparametric estimator based on a linear specification of the 
structural equation and a varying coefficients specification of the first stages that is linear in covariates but nonparametric in the running variable. We have provided an asymptotic analysis for this semiparametric estimator which parallels that of the classical RDD estimator for binary endogenous variables in Hahn, Todd and van der Klaauw (1990). The estimator can be also implemented as a simple TSLS.

\section{A Appendix}

\section{A.1 Theory for Parametric and Semiparametric Estimators}

\section{A.1.1 Proof of Theorem 3.2}

We prove the consistency of the TSLS estimator. Its asymptotic distribution follows standard arguments, which are therefore omitted. Using equation (14) as a first stage identifies the coefficients of the regression of $Y$ on a constant, $X^{*}$ and $Z$, where

$$
X^{*}=\alpha_{0 X}+\alpha_{1 X} T+\alpha_{2 X}^{\prime} Z+\alpha_{3 X}^{\prime} Z T .
$$

By the Frisch-Waugh-Lowell Theorem, the slope of $X^{*}$, say $\beta^{*}$, is given by

$$
\beta^{*}=\left(\mathbb{E}\left[\Pi_{z} \Pi_{z}^{\prime}\right]\right)^{-1} \mathbb{E}\left[\Pi_{z} Y\right]
$$

where $\Pi_{z}=X^{*}-\mathbb{E}\left[X^{*} \mid Z\right]=\mathbb{E}[X \mid T, Z]-\mathbb{E}[X \mid Z]$. We prove that is legitimate to take the inverse of $\mathbb{E}\left[\Pi_{z} \Pi_{z}^{\prime}\right]$ under our conditions, by showing that $\mathbb{E}\left[\Pi_{z} \Pi_{z}^{\prime}\right]$ is invertible if and only if $\mathbb{E}\left[\Delta_{X} \Delta_{X}^{\prime}\right]$ is invertible. To see that, suppose $\mathbb{E}\left[\Delta_{X} \Delta_{X}^{\prime}\right]$ is singular. Then, there exists a $\lambda \neq 0$ such that, a.s.

$$
\mathbb{E}\left[\lambda^{\prime} X \mid T=1, Z\right]=\mathbb{E}\left[\lambda^{\prime} X \mid T=0, Z\right] .
$$

Then,

$$
\mathbb{E}\left[\lambda^{\prime} X \mid T, Z\right]=\mathbb{E}\left[\lambda^{\prime} X \mid Z\right],
$$

and therefore, $\lambda^{\prime} \Pi_{z}=0$ a.s. (i.e. $\mathbb{E}\left[\Pi_{z} \Pi_{z}^{\prime}\right]$ is singular). The reciprocal follows the same arguments.

Then, substituting (13) into $\beta^{*}$, yields that $\beta^{*}=\beta$, thereby proving the consistency of the TSLS for $\beta$.

\section{A.1.2 Theory for Semiparametric RDD}

In this section we establish the asymptotic normality for a generalized version of the TSLS $\tilde{\beta}$ for RDD where a general kernel, not necessarily the uniform kernel (19), is used. For simplicity, we only consider the case where $X$ and $Z$ are univariate. Without loss of generality assume hereinafter that $\bar{w}=0$. We introduce some notation. Let $\varepsilon_{V_{i}}=V_{i}-\mathbb{E}\left[V_{i} \mid W_{i}, Z_{i}\right]$ denote the regression errors for $V=Y$ and $V=X$. We assume that

$$
\mathbb{E}[V \mid Z, W]=: \alpha_{0 V}(W)+\alpha_{1 V}^{\prime}(W) Z
$$

for both $V=Y$ and $V=X$. To simplify notation denote

$$
\lim _{w \downarrow 0} \alpha_{0 V}(w)=\alpha_{0 V}^{+} \quad \lim _{w \downarrow 0} \alpha_{1 V}(w)=\alpha_{1 V}^{+}
$$


and

$$
\lim _{w \uparrow 0} \alpha_{0 V}(w)=\alpha_{0 V}^{-} \quad \lim _{w \uparrow 0} \alpha_{1 V}(w)=\alpha_{1 V}^{-} .
$$

We can then estimate these quantities using local linear regression. Precisely, for $V=X$ or $V=Y$, let $\alpha_{V}^{+}=\left(\alpha_{0 V}^{+}, \alpha_{1 V}^{+\prime}, \alpha_{2 V}^{+}, \alpha_{3 V}^{+\prime}\right)^{\prime}$, and

$$
\widehat{\alpha}_{V}^{+}=\arg \min _{\alpha_{V}^{+}} \sum_{i=1}^{n}\left(V_{i}-\alpha_{0 V}^{+}-\alpha_{1 V}^{+\prime} Z_{i}-\alpha_{2 V}^{+} W_{i}-\alpha_{3 V}^{+\prime} Z_{i} W_{i}\right)^{2} k_{h}\left(W_{i}\right) 1\left(W_{i} \geq 0\right),
$$

where $k_{h}(W)=k(W / h), k$ is a kernel function and $h$ is a bandwidth parameter satisfying some conditions below (Assumption 4). The estimation of $\widehat{\alpha}_{0 V}^{-}$and $\widehat{\alpha}_{1 V}^{-}$for $V=Y$ and $V=X$ is analogous, replacing $1\left(W_{i} \geq 0\right)$ in the minimization problem by $1\left(W_{i}<0\right)$.

We can now apply the arguments of equation (16). Notice that in this setting $\delta_{X i}=\alpha_{0 X}^{+}-\alpha_{0 X}^{-}+$ $\left(\alpha_{1 X}^{+}-\alpha_{1 X}^{-}\right)^{\prime} Z_{i}$ and $\delta_{Y i}=\alpha_{0 Y}^{+}-\alpha_{0 Y}^{-}+\left(\alpha_{1 Y}^{+}-\alpha_{1 Y}^{-}\right)^{\prime} Z_{i}$, and thus we can obtain $\widehat{\delta}_{Y i}$ and $\widehat{\delta}_{X i}$ by substituting the estimated $\alpha$ 's from the previous step. Then, $\tilde{\beta}$ is obtained from the OLS regression of the $\widehat{\delta}_{Y i}$ on the $\widehat{\delta}_{X i}$ (without intercept), and has the closed-form expression

$$
\tilde{\beta}=\left(\sum_{i=1}^{n} \widehat{\delta}_{X i} \widehat{\delta}_{X i}^{\prime}\right)^{-1} \sum_{i=1}^{n} \widehat{\delta}_{X i} \widehat{\delta}_{Y i}
$$

We investigate the asymptotic properties of $\tilde{\beta}$ under the following assumptions, which parallel those of Hahn, Todd and van der Klaauw (1990):

\section{Assumption 4 Suppose that}

1. For $w>0$ and $V=Y$ and $X, \alpha_{0 V}(w)$ and $\alpha_{1 V}(w)$ are twice continuously differentiable. There exists some $M>0$ such that $\dot{\alpha}_{j V}^{+}(w)=\lim _{u \downarrow w} \partial \alpha_{j V}(u) / \partial u$ and $\ddot{\alpha}_{j V}^{+}(w)=\lim _{u \downarrow w} \partial^{2} \alpha_{j V}(u) / \partial u^{2}$ are uniformly bounded on $(0, M]$, for $j=0,1$. Similarly, $\dot{\alpha}_{j V}^{-}(w)=\lim _{u \uparrow w} \partial \alpha_{j V}(u) / \partial u$ and $\ddot{\alpha}_{j V}^{-}(w)=\lim _{u \uparrow w} \partial^{2} \alpha_{j V}(u) / \partial u^{2}$ are uniformly bounded on $[-M, 0)$, for $j=0,1$.

2. Equation (21) holds. The limits $\left(\alpha_{j V}^{+}, \dot{\alpha}_{j V}^{+}, \ddot{\alpha}_{j V}^{+}, \alpha_{j V}^{-}, \dot{\alpha}_{j V}^{-}, \ddot{\alpha}_{j V}^{-}\right)$are well-defined and finite for $j=0,1$ and $V=Y$ and $X$.

3. The density of $W, f(w)$, is continuous and bounded near $w=0$. It is also bounded away from zero near $w=0$.

4. The kernel $k$ is continuous, symmetric and nonnegative-valued with compact support.

5. The functions $\mu_{j}(w)=\mathbb{E}\left[Z^{j} \mid W=w\right], q_{Y j}(w)=\mathbb{E}\left[Z^{2 j} \varepsilon_{Y_{i}}^{2} \mid W=w\right], q_{X j}(w)=\mathbb{E}\left[Z^{2 j} \varepsilon_{X_{i}}^{2} \mid W=w\right]$, $r_{j}(w)=\mathbb{E}\left[Z^{2 j} \varepsilon_{Y_{i}} \varepsilon_{X_{i}} \mid W=w\right], s_{Y j}(w)=\mathbb{E}\left[Z^{3 j} \varepsilon_{Y_{i}}^{3} \mid W=w\right]$ and $s_{X j}(w)=\mathbb{E}\left[Z^{3 j} \varepsilon_{X_{i}}^{3} \mid W=w\right]$ are uniformly bounded near $w=0$, with well-defined and finite left and right limits to $w=0$, for $j=0,1$ and 2 .

6. The bandwidth satisfies $n h^{5} \rightarrow 0$. 
Define the vectors $\widehat{\delta}_{h}=\left(\widehat{\delta}_{0 Y}, \widehat{\delta}_{1 Y}, h \widehat{\delta}_{2 Y}, h \widehat{\delta}_{3 Y}, \widehat{\delta}_{0 X}, \widehat{\delta}_{1 X}, h \widehat{\delta}_{2 X}, h \widehat{\delta}_{3 X}\right)^{\prime}, \delta_{h}=\left(\delta_{0 Y}, \delta_{1 Y}, h \delta_{2 Y}, h \delta_{3 Y}, \delta_{0 X}\right.$, $\left.\delta_{1 X}, h \delta_{2 X}, h \delta_{3 X}\right)^{\prime}$, where $\widehat{\delta}_{j V}=\widehat{\alpha}_{j V}^{+}-\widehat{\alpha}_{j V}^{-}$and $\delta_{j V}=\alpha_{j V}^{+}-\alpha_{j V}^{-}$for $j=0,1,2,3$ and $V=Y$ or $X$. There are two main steps in the proof of asymptotic normality of $\tilde{\beta}$. First, we show that

$$
\begin{aligned}
\tilde{\beta}-\beta_{0} & =\left(E\left[\delta_{X i}^{2}\right]\right)^{-1} E\left[\delta_{X} \tilde{Z}_{i}^{\prime}\right] B_{2}\left(\widehat{\delta}_{h}-\delta_{h}\right)+o_{P}\left((n h)^{-1 / 2}\right) \\
& \equiv Q_{2}\left(\widehat{\delta}_{h}-\delta_{h}\right)+o_{P}\left((n h)^{-1 / 2}\right)
\end{aligned}
$$

where $Q_{2}=\left(E\left[\delta_{X i}^{2}\right]\right)^{-1} E\left[\delta_{X} \tilde{Z}_{i}^{\prime}\right] B_{2}$, and

$$
B_{2}=\left[\begin{array}{cccccccc}
1 & 0 & 0 & 0 & -\beta_{0} & 0 & 0 & 0 \\
0 & 1 & 0 & 0 & 0 & -\beta_{0} & 0 & 0
\end{array}\right]
$$

Second, following similar arguments to those of Hahn, Todd and van der Klaauw (1990) we show in Lemma A.8 that

$$
\sqrt{n h}\left(\widehat{\delta}_{h}-\delta_{h}\right) \rightarrow_{d} N(0, \Omega),
$$

and provide an expression for $\Omega$. The asymptotic normality then follows from (22) and (23).

To prove (22), use $\delta_{Y i}=\beta_{0} \delta_{X i}$ and substitute $\widehat{\delta}_{Y i}=\beta_{0} \widehat{\delta}_{X i}+\widehat{\delta}_{Y i}-\delta_{Y i}-\beta_{0}\left(\widehat{\delta}_{X i}-\delta_{X i}\right)$ in $\tilde{\beta}$ to get

$$
\tilde{\beta}=\beta_{0}+\frac{\sum_{i=1}^{n} \widehat{\delta}_{X i}\left(\widehat{\delta}_{Y i}-\delta_{Y i}\right)}{\sum_{i=1}^{n} \widehat{\delta}_{X i}^{2}}-\beta_{0} \frac{\sum_{i=1}^{n} \widehat{\delta}_{X i}\left(\widehat{\delta}_{X i}-\delta_{X i}\right)}{\sum_{i=1}^{n} \widehat{\delta}_{X i}^{2}} .
$$

By Lemmas A.1-A.8 below, we can further write

$$
\tilde{\beta}-\beta_{0}=\left(E\left[\delta_{X i}^{2}\right]\right)^{-1}\left(\frac{1}{n} \sum_{i=1}^{n} \delta_{X i}\left(\widehat{\delta}_{Y i}-\delta_{Y i}\right)-\frac{\beta_{0}}{n} \sum_{i=1}^{n} \delta_{X i}\left(\widehat{\delta}_{X i}-\delta_{X i}\right)\right)+o_{P}\left((n h)^{-1 / 2}\right) .
$$

Henceforth we focus on the analysis for $\alpha_{V}^{+}$, since that of $\alpha_{V}^{-}$is symmetric. Introduce the notation $f\left(0^{+}\right)=\lim _{w \downarrow 0} f(w)$,

$$
V_{i}^{+}=V_{i}-\alpha_{V}^{+\prime} S_{i} \quad \text { and } \quad k_{i h}^{+}=k_{h}\left(W_{i}\right) 1\left(W_{i} \geq 0\right),
$$

where $S_{i}:=\left(1, Z_{i}, W_{i}, W_{i} Z_{i}\right)^{\prime}$ and $S_{i h}:=\left(1, Z_{i}, W_{i} / h, Z_{i} W_{i} / h\right)^{\prime}$. Then, with this notation we can write the OLS problem for $\alpha_{V}^{+}$as

$$
\min _{\alpha} \sum_{i=1}^{n}\left(V_{i}^{+}-\left(\alpha-\alpha_{V}^{+}\right)^{\prime} S_{i}\right)^{2} k_{i h}^{+}
$$

whose first order condition yields

$$
\left[\begin{array}{c}
\widehat{\alpha}_{0 V}^{+}-\alpha_{0 V}^{+} \\
\widehat{\alpha}_{1 V}^{+}-\alpha_{1 V}^{+} \\
h\left(\widehat{\alpha}_{2 V}^{+}-\alpha_{2 V}^{+}\right) \\
h\left(\widehat{\alpha}_{3 V}^{+}-\alpha_{3 V}^{+}\right)
\end{array}\right]=\left(\sum_{i=1}^{n} S_{i h} S_{i h}^{\prime} k_{i h}^{+}\right)^{-1}\left(\sum_{i=1}^{n} S_{i h} V_{i}^{+} k_{i h}^{+}\right)
$$

Based on this expression we analyze the asymptotic properties of the local linear estimators. All the Lemmas below make use of Assumptions 2, 3 and 4. 


\section{Lemma A.1 (Denominator)}

$$
\frac{1}{n h} \sum_{i=1}^{n} S_{i h} S_{i h}^{\prime} k_{i h}^{+} \rightarrow_{p} f\left(0^{+}\right) \Gamma_{d}
$$

where

$$
\begin{aligned}
& \Gamma_{d}=\left[\begin{array}{cccc}
\gamma_{0} & \gamma_{0} \mu_{1}^{+} & \gamma_{1} & \gamma_{1} \mu_{1}^{+} \\
\gamma_{0} \mu_{1}^{+} & \gamma_{0} \mu_{2}^{+} & \gamma_{1} \mu_{1}^{+} & \gamma_{1} \mu_{2}^{+} \\
\gamma_{1} & \gamma_{1} \mu_{1}^{+} & \gamma_{2} & \gamma_{2} \mu_{1}^{+} \\
\gamma_{1} \mu_{1}^{+} & \gamma_{1} \mu_{2}^{+} & \gamma_{2} \mu_{1}^{+} & \gamma_{2} \mu_{2}^{+}
\end{array}\right] \\
& \gamma_{l}=\int_{0}^{\infty} u^{l} k(u) d u \quad \text { and } \quad \mu_{j}^{+}=\lim _{w \downarrow 0} \mathbb{E}\left[Z^{j} \mid W=w\right] .
\end{aligned}
$$

Proof. Let

$$
\theta_{l j}=\frac{1}{n h} \sum_{i=1}^{n}\left(\frac{W_{i}}{h}\right)^{l} Z_{i}^{j} k_{i h}^{+}, \quad l, j=0,1,2 .
$$

Then, by the change of variables $u=w / h$,

$$
\begin{aligned}
\mathbb{E}\left[\theta_{l j}\right] & =h^{-1} \mathbb{E}\left[\left(\frac{W_{i}}{h}\right)^{l} Z_{i}^{j} k_{i h}^{+}\right] \\
& =\int_{0}^{\infty} u^{l} k(u) \mu_{j}(u h) f(u h) d u \\
& =\mu_{j}^{+} f\left(0^{+}\right) \gamma_{l}+o(1),
\end{aligned}
$$

where $\mu_{j}(w)=\mathbb{E}\left[Z^{j} \mid W=w\right]$ and the convergence follows by the Dominated Convergence theorem. As for the variance

$$
\begin{aligned}
\operatorname{Var}\left(\theta_{l j}\right) & \leq\left(n h^{2}\right)^{-1} \mathbb{E}\left[\left(\frac{W_{i}}{h}\right)^{2 l} Z_{i}^{2 j} k_{i h}^{+2}\right] \\
& =(n h)^{-1} \int_{0}^{\infty} u^{2 l} k^{2}(u) \mu_{2 j}(u h) f(u h) d u \\
& =o(1),
\end{aligned}
$$

again by the Dominated Convergence theorem.

We now consider the asymptotic behaviour of the numerator. Define the function

$$
\begin{aligned}
\zeta_{V}(w, z) & =\alpha_{0 V}(w)+\alpha_{1 V}(w) z-\alpha_{0 V}^{+}+\alpha_{1 V}^{+} z \\
& -\left(\dot{\alpha}_{0 V}^{+}+\dot{\alpha}_{1 V}^{+} z\right) w-\frac{1}{2}\left(\ddot{\alpha}_{0 V}^{+}+\ddot{\alpha}_{1 V}^{+} z\right) w^{2},
\end{aligned}
$$

where $\dot{\alpha}_{0 V}^{+}=\lim _{w \downarrow 0} \partial \alpha_{0 V}(w) / \partial w$ and $\ddot{\alpha}_{0 V}^{+}=\lim _{w \downarrow 0} \partial^{2} \alpha_{0 V}(w) / \partial w^{2}$, and similarly for $\alpha_{1 V}$. Note that $\dot{\alpha}_{0 V}^{+}=\alpha_{2 V}^{+}$and $\ddot{\alpha}_{0 V}^{+}=\alpha_{3 V}^{+}$and observe that

$$
\sup _{0<w<M h}\left|\zeta_{V}(w, z)\right|=o\left(h^{2}\right)(1+|Z|) .
$$




\section{Lemma A.2 (Numerator: Expectation)}

$$
\mathbb{E}\left[\frac{1}{n h} \sum_{i=1}^{n} S_{i h} V_{i}^{+} k_{i h}^{+}\right] \rightarrow_{p} \frac{1}{2} f\left(0^{+}\right) h^{2}\left(b_{V}^{+}+o(1)\right),
$$

where

$$
b_{V}^{+}=\left[\begin{array}{c}
\gamma_{2}\left(\ddot{\alpha}_{0 V}^{+}+\ddot{\alpha}_{0 V}^{+} \mu_{1}^{+}\right) \\
\gamma_{2}\left(\ddot{\alpha}_{0 V}^{+} \mu_{1}^{+}+\ddot{\alpha}_{0 V}^{+} \mu_{2}^{+}\right) \\
\gamma_{3}\left(\ddot{\alpha}_{0 V}^{+}+\ddot{\alpha}_{0 V}^{+} \mu_{1}^{+}\right) \\
\gamma_{3}\left(\ddot{\alpha}_{0 V}^{+} \mu_{1}^{+}+\ddot{\alpha}_{0 V}^{+} \mu_{2}^{+}\right)
\end{array}\right]
$$

Proof. Let

$$
u_{l j}=\frac{1}{n h} \sum_{i=1}^{n}\left(\frac{W_{i}}{h}\right)^{l} Z_{i}^{j} V_{i}^{+} k_{i h}^{+}, \quad l, j=0,1 .
$$

Then, write

$$
\begin{aligned}
\mathbb{E}\left[u_{l j}\right] & =h^{-1} \mathbb{E}\left[\left(\frac{W_{i}}{h}\right)^{l} Z_{i}^{j} V_{i}^{+} k_{i h}^{+}\right] \\
& =h^{-1} \mathbb{E}\left[\left(\frac{W_{i}}{h}\right)^{l} Z_{i}^{j}\left(\frac{1}{2}\left(\ddot{\alpha}_{0 V}\left(0^{+}\right)+\ddot{\alpha}_{1 V}\left(0^{+}\right) Z_{i}\right) W_{i}^{2}+\zeta_{V}\left(W_{i}, Z_{i}\right)\right) k_{i h}^{+}\right] \\
& =h^{-1} \frac{1}{2} \ddot{\alpha}_{0 V}\left(0^{+}\right) \mathbb{E}\left[\left(\frac{W_{i}}{h}\right)^{l} Z_{i}^{j} W_{i}^{2} k_{i h}^{+}\right]+h^{-1} \frac{1}{2} \ddot{\alpha}_{1 V}\left(0^{+}\right) \mathbb{E}\left[\left(\frac{W_{i}}{h}\right)^{l} Z_{i}^{j+1} W_{i}^{2} k_{i h}^{+}\right] \\
& +h^{-1} \mathbb{E}\left[\left(\frac{W_{i}}{h}\right)^{l} Z_{i}^{j} \zeta_{V}\left(W_{i}, Z_{i}\right) k_{i h}^{+}\right] .
\end{aligned}
$$

By the change of variables $u=w / h$,

$$
\begin{aligned}
h^{-1} \mathbb{E}\left[\left(\frac{W_{i}}{h}\right)^{l} Z_{i}^{j} W_{i}^{2} k_{i h}^{+}\right] & =h^{2} \int_{0}^{\infty} u^{l+2} k(u) \mu_{j}(u h) f(u h) d u \\
& =h^{2} \mu_{j}^{+} f\left(0^{+}\right) \gamma_{l+2}+o(1),
\end{aligned}
$$

and similarly

$$
h^{-1} \mathbb{E}\left[\left(\frac{W_{i}}{h}\right)^{l} Z_{i}^{j+1} W_{i}^{2} k_{i h}^{+}\right]=h^{2} \mu_{j+1}^{+} f\left(0^{+}\right) \gamma_{l+2}+o(1) .
$$

On the other hand, assume without loss of generality that $[-M, M]$ contains the support of $k$, so that

$$
h^{-1} \mathbb{E}\left[\left(\frac{W_{i}}{h}\right)^{l} Z_{i}^{j} \zeta_{V}\left(W_{i}, Z_{i}\right) k_{i h}^{+}\right]=o\left(h^{2}\right) .
$$

\section{Lemma A.3 (Numerator: Conditional Expectation)}

$$
\frac{1}{n h} \sum_{i=1}^{n} \mathbb{E}\left[S_{i h} V_{i}^{+} k_{i h}^{+} \mid W_{i}, Z_{i}\right]=\frac{1}{n h} \sum_{i=1}^{n} \mathbb{E}\left[S_{i h} V_{i}^{+} k_{i h}^{+}\right]+o_{p}\left(h^{2}\right) .
$$


Proof. We have

$$
\begin{aligned}
\frac{1}{n h} \sum_{i=1}^{n} \mathbb{E}\left[S_{i h} V_{i}^{+} k_{i h}^{+} \mid W_{i}, Z_{i}\right] & =\frac{1}{n h} \sum_{i=1}^{n} S_{i h} k_{i h}^{+}\left(\frac{1}{2}\left(\ddot{\alpha}_{0 V}\left(0^{+}\right)+\ddot{\alpha}_{1 V}\left(0^{+}\right) Z_{i}\right) W_{i}^{2}+\zeta_{V}\left(W_{i}, Z_{i}\right)\right) \\
& =\frac{1}{2} \ddot{\alpha}_{0 V}\left(0^{+}\right) \frac{1}{n h} \sum_{i=1}^{n} S_{i h} k_{i h}^{+} W_{i}^{2}+\frac{1}{2} \ddot{\alpha}_{1 V}\left(0^{+}\right) \frac{1}{n h} \sum_{i=1}^{n} S_{i h} k_{i h}^{+} Z_{i} W_{i}^{2} \\
& +\frac{1}{n h} \sum_{i=1}^{n} S_{i h} k_{i h}^{+} \zeta_{V}\left(W_{i}, Z_{i}\right) .
\end{aligned}
$$

Observe that

$$
\begin{aligned}
\operatorname{Var}\left(\frac{1}{n h} \sum_{i=1}^{n} S_{i h} k_{i h}^{+} W_{i}^{2}\right) & =\left(n h^{2}\right)^{-1} \operatorname{Var}\left(S_{i h} k_{i h}^{+} W_{i}^{2}\right) \\
& \leq C(n h)^{-1} h^{-1} \mathbb{E}\left[S_{i h} S_{i h}^{\prime} k_{i h}^{+2} W_{i}^{4}\right] \\
& =O\left((n h)^{-1} h^{4}\right) \\
& =o(1)
\end{aligned}
$$

since for $l, j=0,1,2$

$$
\begin{aligned}
h^{-1} \mathbb{E}\left[\left(\frac{W_{i}}{h}\right)^{l} Z_{i}^{j} k_{i h}^{+2} W_{i}^{4}\right] & =h^{4} \int_{0}^{\infty} u^{l} k^{2}(u) \mu_{j}(u h) f(u h) d u \\
& =h^{4} \mu_{j}^{+} f\left(0^{+}\right) v_{l}+o(1),
\end{aligned}
$$

where

$$
v_{l}=\int_{0}^{\infty} u^{l} k^{2}(u) d u
$$

Similarly,

$$
\operatorname{Var}\left(\frac{1}{n h} \sum_{i=1}^{n} S_{i h} k_{i h}^{+} Z_{i} W_{i}^{2}\right)=o(1)
$$

and

$$
\operatorname{Var}\left(\frac{1}{n h} \sum_{i=1}^{n} S_{i h} k_{i h}^{+} \zeta_{V}\left(W_{i}, Z_{i}\right)\right)=o(1)
$$

Note that

$$
\frac{1}{n h} \sum_{i=1}^{n} S_{i h} k_{i h}^{+}\left(V_{i}^{+}-\mathbb{E}\left[V_{i}^{+} \mid W_{i}, Z_{i}\right]\right)=\frac{1}{n h} \sum_{i=1}^{n} S_{i h} k_{i h}^{+} \varepsilon_{V_{i}}
$$

where $\varepsilon_{V_{i}}=V_{i}-\mathbb{E}\left[V_{i} \mid W_{i}, Z_{i}\right]$ denotes the regression error. Then, we have the following result.

\section{Lemma A.4 (Numerator: Conditional Variance)}

$$
\operatorname{Var}\left(\frac{1}{n h} \sum_{i=1}^{n} S_{i h} k_{i h}^{+}\left(V_{i}^{+}-\mathbb{E}\left[V_{i}^{+} \mid W_{i}, Z_{i}\right]\right)\right)=\frac{1}{n h} f\left(0^{+}\right) \Sigma_{V^{+}}+o(1),
$$


where

$$
\begin{gathered}
\Sigma_{V^{+}}=\left[\begin{array}{cccc}
v_{0} & v_{0} q_{1}^{+} & v_{2} & v_{2} q_{1}^{+} \\
v_{0} q_{1}^{+} & v_{0} q_{2}^{+} & v_{2} q_{1}^{+} & v_{2} q_{2}^{+} \\
v_{2} & v_{2} q_{1}^{+} & v_{4} & v_{4} q_{1}^{+} \\
v_{2} q_{1}^{+} & v_{2} q_{2}^{+} & v_{4} q_{1}^{+} & v_{4} q_{2}^{+}
\end{array}\right], \\
v_{l}=\int_{0}^{\infty} u^{l} k^{2}(u) d u \text { and } q_{j}^{+}=\lim _{w \downarrow 0} \mathbb{E}\left[Z^{2 j} \varepsilon_{V_{i}}^{2} \mid W=w\right] .
\end{gathered}
$$

Proof. Consider the generic term, for $l, j=0,1$

$$
\frac{1}{n h} \sum_{i=1}^{n}\left(\frac{W_{i}}{h}\right)^{l} Z_{i}^{j} k_{i h}^{+} \varepsilon_{V_{i}}
$$

and its variance, which equals

$$
\begin{aligned}
(n h)^{-1} h^{-1} \mathbb{E}\left[\left(\frac{W_{i}}{h}\right)^{2 l} Z_{i}^{2 j} k_{i h}^{+2} \varepsilon_{V_{i}}^{2}\right] & =(n h)^{-1} \int_{0}^{\infty} u^{2 l} k^{2}(u) q_{j}(u h) f(u h) d u \\
& =(n h)^{-1} f\left(0^{+}\right) q_{j}^{+} v_{2 l}+o(1)
\end{aligned}
$$

where $q_{j}(w)=\mathbb{E}\left[Z^{2 j} \varepsilon_{V_{i}}^{2} \mid W=w\right]$.

\section{Lemma A.5 (Numerator: Conditional Covariance)}

$$
\operatorname{Cov}\left(\frac{1}{n h} \sum_{i=1}^{n} S_{i h} k_{i h}^{+} \varepsilon_{Y_{i}}, \frac{1}{n h} \sum_{i=1}^{n} S_{i h} k_{i h}^{+} \varepsilon_{X_{i}}\right)=\frac{1}{n h} f\left(0^{+}\right) \Sigma_{Y X^{+}}+o(1),
$$

where

$$
\Sigma_{Y X^{+}}=\left[\begin{array}{cccc}
v_{0} & v_{0} r_{1}^{+} & v_{2} & v_{2} r_{1}^{+} \\
v_{0} r_{1}^{+} & v_{0} r_{2}^{+} & v_{2} r_{1}^{+} & v_{2} r_{2}^{+} \\
v_{2} & v_{2} r_{1}^{+} & v_{4} & v_{4} r_{1}^{+} \\
v_{2} r_{1}^{+} & v_{2} r_{2}^{+} & v_{4} r_{1}^{+} & v_{4} r_{2}^{+}
\end{array}\right]
$$

and

$$
r_{j}^{+}=\lim _{w \downarrow 0} \mathbb{E}\left[Z^{2 j} \varepsilon_{Y_{i}} \varepsilon_{X_{i}} \mid W=w\right] .
$$

Proof. The proof is analogous to the previous Lemma, and hence it is omitted.

\section{Lemma A.6 (Numerator: Conditional CLT)}

$$
(n h)^{-1 / 2} \sum_{i=1}^{n}\left(\begin{array}{c}
S_{i h} k_{i h}^{+} \varepsilon_{Y_{i}} \\
S_{i h} k_{i h}^{+} \varepsilon_{X_{i}}
\end{array}\right) \rightarrow_{d} f\left(0^{+}\right) N\left(0,\left[\begin{array}{cc}
\Sigma_{Y^{+}} & \Sigma_{Y^{+}} \\
\Sigma_{Y X^{+}} & \Sigma_{X^{+}}
\end{array}\right]\right) .
$$

Proof. Consider a generic term for $l, j=0,1$

$$
\frac{1}{\sqrt{n h}} \sum_{i=1}^{n}\left(\frac{W_{i}}{h}\right)^{l} Z_{i}^{j} k_{i h}^{+} \varepsilon_{V_{i}} .
$$


We apply Lyapounov with third absolute moment. By the lemma on the asymptotic variance, we need to establish

$$
(n h)^{-1 / 2} h^{-1} \mathbb{E}\left[\left(\frac{W_{i}}{h}\right)^{3 l} Z_{i}^{3 j} k_{i h}^{+3} \varepsilon_{V_{i}}^{3}\right]=o(1) .
$$

But note that, defining $s_{j}(w)=\mathbb{E}\left[Z^{3 j} \varepsilon_{V_{i}}^{3} \mid W=w\right]$,

$$
\begin{aligned}
h^{-1} \mathbb{E}\left[\left(\frac{W_{i}}{h}\right)^{3 l} Z_{i}^{3 j} k_{i h}^{+3} \varepsilon_{V_{i}}^{3}\right] & =\int_{0}^{\infty} u^{3 l} k^{3}(u) s_{j}(u h) f(u h) d u \\
& =O(1) .
\end{aligned}
$$

\section{Lemma A.7 (Numerator: Unconditional CLT)}

$$
(n h)^{-1 / 2} \sum_{i=1}^{n}\left(\begin{array}{c}
S_{i h} k_{i h}^{+} Y_{i}^{+} \\
S_{i h} k_{i h}^{+} X_{i}^{+}
\end{array}\right)-\frac{(n h)^{1 / 2} h^{2}}{2} f\left(0^{+}\right)\left(\begin{array}{c}
b_{Y}^{+} \\
b_{X}^{+}
\end{array}\right) \rightarrow_{d} f^{1 / 2}\left(0^{+}\right) N\left(0,\left[\begin{array}{cc}
\Sigma_{Y^{+}} & \Sigma_{Y^{+}} \\
\Sigma_{Y X^{+}} & \Sigma_{X^{+}}
\end{array}\right]\right) .
$$

Proof. It follows from previous Lemmas.

Denote

$$
\widehat{\alpha}_{h}^{+}-\alpha_{h}^{+}=\left[\begin{array}{c}
\widehat{\alpha}_{0 Y}^{+}-\alpha_{0 Y}^{+} \\
\widehat{\alpha}_{1 Y}^{+}-\alpha_{1 Y}^{+} \\
h\left(\widehat{\alpha}_{2 Y}^{+}-\alpha_{2 Y}^{+}\right) \\
h\left(\widehat{\alpha}_{3 Y}^{+}-\alpha_{3 Y}^{+}\right) \\
\widehat{\alpha}_{0 X}^{+}-\alpha_{0 X}^{+} \\
\widehat{\alpha}_{1 X}^{+}-\alpha_{1 X}^{+} \\
h\left(\widehat{\alpha}_{2 X}^{+}-\alpha_{2 X}^{+}\right) \\
h\left(\widehat{\alpha}_{3 X}^{+}-\alpha_{3 X}^{+}\right)
\end{array}\right] \quad \widehat{\alpha}_{h}^{-}-\alpha_{h}^{-}=\left[\begin{array}{c}
\widehat{\alpha}_{0 Y}^{-}-\alpha_{0 Y}^{-} \\
\widehat{\alpha}_{1 Y}^{-}-\alpha_{1 Y}^{-} \\
h\left(\widehat{\alpha}_{2 Y}^{-}-\alpha_{2 Y}^{-}\right) \\
h\left(\widehat{\alpha}_{3 Y}^{-}-\alpha_{3 Y}^{-}\right) \\
\widehat{\alpha}_{0 X}^{-}-\alpha_{0 X}^{-} \\
\widehat{\alpha}_{1 X}^{-}-\alpha_{1 X}^{-} \\
h\left(\widehat{\alpha}_{2 X}^{-}-\alpha_{2 X}^{-}\right) \\
h\left(\widehat{\alpha}_{3 X}^{-}-\alpha_{3 X}^{-}\right)
\end{array}\right]
$$

and

$$
\Sigma_{+}=\left[\begin{array}{cc}
\Sigma_{Y^{+}} & \Sigma_{Y^{+}} \\
\Sigma_{Y^{+}} & \Sigma_{X^{+}}
\end{array}\right] \quad \Sigma_{-}=\left[\begin{array}{cc}
\Sigma_{Y^{-}} & \Sigma_{Y X^{-}} \\
\Sigma_{Y^{-}} & \Sigma_{X^{-}}
\end{array}\right],
$$

where the definition of $\Sigma_{-}$is like $\Sigma_{+}$but with limits to the left of $w=0$.

\section{Lemma A.8 (Joint CLT)}

$$
\begin{aligned}
& \sqrt{n h}\left(\widehat{\alpha}_{h}^{+}-\alpha_{h}^{+}-\widehat{\alpha}_{h}^{-}+\alpha_{h}^{-}\right)-\frac{(n h)^{1 / 2} h^{2}}{2}\left[\begin{array}{cc}
\Gamma_{d}^{-1} & 0 \\
0 & \Gamma_{d}^{-1}
\end{array}\right]\left(\begin{array}{c}
b_{Y}^{+}-b_{Y}^{-} \\
b_{X}^{+}-b_{X}^{-}
\end{array}\right) \\
& \rightarrow_{d} f^{-1 / 2}\left(0^{+}\right)\left[\begin{array}{cc}
\Gamma_{d}^{-1} & 0 \\
0 & \Gamma_{d}^{-1}
\end{array}\right] N\left(0, \Sigma_{+}+\Sigma_{-}\right) .
\end{aligned}
$$

Proof. It follows from previous Lemmas and the asymptotic independence of $(n h)^{-1 / 2}\left(\widehat{\alpha}_{h}^{+}-\alpha_{h}^{+}\right)$and $(n h)^{-1 / 2}\left(\widehat{\alpha}_{h}^{-}-\alpha_{h}^{-}\right)$. 
Define

$$
\Omega=\left(\Sigma_{+}+\Sigma_{-}\right) .
$$

Proof of the Theorem 4.1:. From (22),

$$
\sqrt{n h}\left(\tilde{\beta}-\beta_{0}\right)=Q_{2} \sqrt{n h}\left(\widehat{\delta}_{h}-\delta_{h}\right)+o_{P}(1) .
$$

Then, apply the previous Lemma and Slutsky's Lemma.

\section{A.2 Nonparametric Estimators}

\section{A.2.1 Binary Instrument Case}

We first introduce some notation that will be used throughout this Section. Henceforth, $A^{\prime}, \operatorname{rank}(A)$, $A^{-}, \operatorname{tr}(A)$ and $|A|:=\left(\operatorname{tr}\left(A^{\prime} A\right)\right)^{1 / 2}$ denote the transpose, rank, Moore-Penrose generalized inverse, trace and the Euclidean norm of a matrix $A$, respectively. The symbol $:=$ denotes definitional relation. For generic random vectors $\zeta$ and $\xi$, let $F_{\zeta}$ and $F_{\zeta / \xi}$ be the cumulative distribution function (cdf) and conditional cdf of $\zeta$ and $\zeta$ given $\xi$, respectively. Denote the corresponding densities with respect to a $\sigma$ finite measure $\mu(x)$ by $f_{\zeta}$ and $f_{\zeta / \xi}$. Unless otherwise stated, the underlying measure will be the Lebesgue measure. Let $\mathcal{S}_{\zeta}$ denote the support of $\zeta$. Let $L_{2}(\zeta)$ denote the Hilbert space with inner product $\langle h, g\rangle:=\int f(x) g(x) d F_{\zeta}(x)$ and the corresponding norm $\|g\|_{2}^{2}:=\langle g, g\rangle$. Henceforth, sometimes we drop the domain of integration for simplicity of notation. For a linear operator $K: L_{2}(X) \rightarrow L_{2}(Y)$, denote the subspaces $\mathcal{R}(K):=\left\{f \in L_{2}(Y): \exists s \in L_{2}(X), K s=f\right\}$ and $\mathcal{N}(K):=\left\{f \in L_{2}(X): K f=0\right\}$. Let $\mathcal{D}(K)$ denote the domain of definition of $K$. Let $K^{*}$ denote the adjoint operator of $K$. We will use some basic results from operator theory and Hilbert spaces. See Carrasco, Florens and Renault (2006) for an excellent review of these results.

Equation (7) provides an integral equation of the first kind that can be used for estimating $g$. Similar estimators have been proposed before in Newey and Powell (2003), Hall and Horowitz (2005), Blundell, Chen and Kristensen (2007), Darolles, Fan, Florens and Renault (2011), Horowitz (2011) and Chen and Pouzo (2012), among others. Here, we follow closely Blundell, Chen and Kristensen (2007). Although, strictly speaking, our model is not given by a conditional moment restriction on a unique set of covariates, we can easily adapt the existing results to make them applicable in our setting. For simplicity, we focus here on the univariate $Z$ and $X$ case.

There are many nonparametric methods that can be used to estimate $m$ and $A$. Here we follow Blundell, Chen and Kristensen (2007) and use a sieve OLS estimator (SLS), see also Ai and Chen (2003) and Newey and Powell (2003). Optimally weighted estimators can be obtained applying ideas in Blundell, Chen and Kristensen (2007). We assume we have a random (i.e. independent and identically distributed, in short iid) sample $\left\{\left(Y_{i}, X_{i}, Z_{i}, T_{i}\right)\right\}_{i=1}^{n}$ of size $n \geq 1$, with the same distribution as the fourth-dimensional vector $(Y, X, Z, T)$. We assume $g$ is in a suitable space of smooth functions. Suppose $\mathcal{S}_{X}$ is a bounded interval of $\mathbb{R}$, with non-empty interior. For any smooth function $h: \mathcal{S}_{X} \subset \mathbb{R} \rightarrow \mathbb{R}$ and some $r>0$, let $[r]$ be the largest integer smaller than $r$, and

$$
\|h\|_{\infty, r}:=\max _{j \leq \underline{\eta}} \sup _{x \in \mathcal{S}_{X}}\left|\nabla^{j} h(x)\right|+\sup _{x \neq x^{\prime}} \frac{\left|\nabla^{[r]} h(x)-\nabla^{[r]} h\left(x^{\prime}\right)\right|}{\left|x-x^{\prime}\right|^{r-\underline{[r]}}} .
$$


Further, let $C_{c}^{r}\left(\mathcal{S}_{X}\right)$ be the set of all continuous functions $h$ with $\|h\|_{\infty, r} \leq c$. Since the constant $c$ is irrelevant for our results, we drop the dependence on $c$ and denote $C^{r}\left(\mathcal{S}_{X}\right)$. We shall assume that $g \in C^{r}\left(\mathcal{S}_{X}\right)$ for some $r$ and approximate $C^{r}\left(\mathcal{S}_{X}\right)$ with a sieve space $\mathcal{G}_{n}$ satisfying some conditions below. Define $k_{n}=\operatorname{dim}\left(\mathcal{G}_{n}\right)$. Given an integer $s>0$ define the Sobolev norm $\|h\|_{s}^{2}:=\sum_{l=0}^{s}\left\|h^{(s)}\right\|_{2}^{2}$, where $h^{(s)}(x):=\partial^{s} h(x) / \partial x^{s}$, with $h^{(0)} \equiv h$.

We approximate $m_{t}(z) \equiv m(z, t):=\mathbb{E}[Y \mid Z=z, T=t]$ by the function $\tilde{m}(z, t)=: \sum_{j \in \mathcal{J}_{n}} m_{t j} p_{0 j}(z, t)$, where $p_{0 j}$ are some known basis functions and $J_{n}:=\#\left(\mathcal{J}_{n}\right) \rightarrow \infty$ as $n \rightarrow \infty$. We write $\tilde{m}(z, t)=$ $p^{J_{n}}(z, t)^{\prime} m^{J_{n}}(t)$, where $p^{J_{n}}(z, t)=\left(p_{01}(z, t), \ldots, p_{0 J_{n}}(z, t)\right)^{\prime}$ and $m^{J_{n}}(t)=\left(m_{t 1}, \ldots, m_{t J_{n}}\right)$. Define $P:=$ $\left(p^{J_{n}}\left(Z_{1}, T_{1}\right), \ldots, p^{J_{n}}\left(Z_{n}, T_{n}\right)\right)^{\prime}$. Then, the SLS is

$$
\widehat{m}(z, t)=p^{J_{n}}(z, t)^{\prime}\left(P^{\prime} P\right)^{-} \sum_{i=1}^{n} p^{J_{n}}\left(Z_{i}, T_{i}\right) Y_{i} .
$$

More precisely, we take $p^{J_{n}}(z, t)=\left(B^{J_{2 n}}(z), t \times B^{J_{2 n}}(z)\right)$, where $B^{J_{2 n}}(z)$ is a $J_{2 n} \times 1$ vector of univariate B-splines or polynomial splines and $J_{n}=2 J_{2 n}$. We define $\widehat{m}(z):=\widehat{m}(z, 1)-\widehat{m}(z, 0)$.

Similarly, for a fixed $g$, we consider the sieve estimator of $A g$ as $\widehat{A} g=\widehat{A}_{1} g-\widehat{A}_{0} g$, where

$$
\widehat{A}_{t} g=p^{J_{n}}(z, t)^{\prime}\left(P^{\prime} P\right)^{-} \sum_{i=1}^{n} p^{J_{n}}\left(Z_{i}, T_{i}\right) g\left(X_{i}\right) .
$$

Finally, the SLS for $g$ is given by the solution of

$$
\widehat{g}_{n}=\arg \min _{g \in \mathcal{G}_{n}} \frac{1}{n} \sum_{i=1}^{n}\left(\widehat{m}\left(Z_{i}\right)-\widehat{A} g\left(Z_{i}\right)\right)^{2} .
$$

We assume the sieve space $\mathcal{G}_{n}$ is of the form

$$
\begin{aligned}
\mathcal{G}_{n} & =\left\{g_{n}: \mathcal{S}_{X} \rightarrow \mathbb{R}, \sup _{x}\left|g_{n}(x)\right|<c, \sup _{x}\left|\nabla^{[r]} g_{n}(x)\right|<c\right. \\
g_{n}(x) & \left.=\psi^{k_{n}}(x)^{\prime} \Pi, g_{n}\left(x_{0}\right)=0\right\}
\end{aligned}
$$

where $\psi^{k_{n}}(\cdot)$ is a $k_{n} \times 1$ vector of known basis that are at least $\gamma=([r]+1)$ times differentiable and $\Pi$ is a $k_{n} \times 1$ vector of coefficients to be estimated. In the application we use B-splines for $\psi^{k_{n}}$. Blundell, Chen and Kristensen (2007) discussed practical ways to incorporate the constraints into the computation of $\widehat{g}_{n}$. For large samples the unconstrained estimator performs well. Note that $g_{n}\left(x_{0}\right)=0$ is a normalization restriction (location), where $x_{0}$ is an arbitrary point in $\mathcal{S}_{X}$.

The following sieve measure of ill-posedness plays a crucial role in the asymptotic theory of sieve estimators, see Blundell, Chen and Kristensen (2007),

$$
\tau_{n}:=\sup _{g \in \mathcal{G}_{n}} \frac{\|g\|}{\left\|\left(A^{*} A\right)^{1 / 2} g\right\|} .
$$

Consider the following assumptions, which are the same as in Blundell, Chen and Kristensen (2007), and therefore are discussed extensively there.

\section{Assumption 5 Suppose that}


1. The data $\left\{\left(Y_{i}, X_{i}, Z_{i}, T_{i}\right)\right\}_{i=1}^{n}$ are iid and Assumption 2 holds.

2. (i) $g \in C^{r}\left(\mathcal{S}_{X}\right)$ for $r>1 / 2$; (ii) $\mathbb{E}\left[|X|^{2 a}\right]<\infty$ for some $a>r$.

3. For $t=1,2, m_{t} \in C^{r_{m}}\left(\mathcal{S}_{Z}\right)$ with $r_{m}>1 / 2$ and $\mathbb{E}\left[g_{n}(X) \mid Z=\cdot, T=t\right] \in C^{r_{m}}\left(\mathcal{S}_{Z}\right)$ for any $g_{n} \in \mathcal{G}_{n}$.

4. (i) The smallest and the largest eigenvalues of $\mathbb{E}\left[B^{J_{2 n}}(Z) \times B^{J_{2 n}}(Z)^{\prime}\right]$ are bounded and bounded away from zero for each $J_{2 n}$; (ii) $B^{J_{2 n}}(Z)$ is a B-spline basis of order $\gamma>r_{m}>1 / 2$; (iii) the density of $Z$ is continuous, bounded, and bounded away from zero over its support $\mathcal{S}_{Z}$, which is a compact interval with non-empty interior.

5. (i) $k_{n} \rightarrow \infty, J_{2 n} / n \rightarrow 0$; (ii) $\lim _{n \rightarrow \infty}\left(J_{2 n} / k_{n}\right)=c_{0}>1$ and $\lim _{n \rightarrow \infty}\left(k_{n}^{2} / n\right)=0$.

6. There is $g_{n} \in \mathcal{G}_{n}$ such that $\tau_{n}^{2}\left\|A\left(g-g_{n}\right)\right\|^{2} \leq C\left\|g-g_{n}\right\|^{2}$.

The following Theorem establishes rates for $\left\|\widehat{g}_{n}-g\right\|$. Its proof is the same as that of Theorem 2 in Blundell, Chen and Kristensen (2007), hence it is omitted.

Theorem A.9 Let Assumption 5 hold. Then,

$$
\left\|\widehat{g}_{n}-g\right\|=O_{P}\left(k_{n}^{-r}+\tau_{n} \times \sqrt{\frac{k_{n}}{n}}\right) .
$$

\section{A.2.2 RDD Case}

The estimator in the RDD case is also a SLS for $g$ given by the solution of

$$
\widehat{g}_{n}=\arg \min _{g \in \mathcal{G}_{n}} \frac{1}{n} \sum_{i=1}^{n}\left(\widehat{m}\left(Z_{i}\right)-\widehat{A} g\left(Z_{i}\right)\right)^{2},
$$

where now $\widehat{m}$ and $\widehat{A}$ are estimated by local linear kernel estimators. For the sake of space, we only consider the univariate case for $X$ and $Z$, and provide only a sketch of the arguments to avoid repetition with the existing literature. Similarly as before, we write $\widehat{m}(z):=\widehat{m}_{+}(z)-\widehat{m}_{-}(z)$ and $\widehat{A} g=\widehat{A}_{+} g-\widehat{A}_{-} g$, where $\widehat{m}_{+}(z)=\widehat{a}$ in the solution to the least squares problem

$$
\left(\widehat{a}, \widehat{b}_{0}, \widehat{b}_{1}\right)=\arg \min _{a_{0}, b_{0}, b_{1}} \sum_{i=1}^{n}\left(Y_{i}-a_{0}-b_{0} W-b_{1}\left(Z_{i}-z\right)\right)^{2} k_{h}\left(W_{i}\right) k_{h}\left(Z_{i}-z\right) 1(W \geq 0),
$$

where $k_{h}\left(z_{l}\right)=h^{-1} k\left(z_{l} / h\right), k(\cdot)$ is a kernel function, and $h$ denotes a bandwidth parameter satisfying regularity conditions described below. Similarly, $\widehat{m}_{-}(z)=\widehat{a}$ in

$$
\left(\widehat{a}, \widehat{b}_{0}, \widehat{b}_{1}\right)=\arg \min _{a_{0}, b_{0}, b_{1}} \sum_{i=1}^{n}\left(Y_{i}-a_{0}-b_{0} W-b_{1}\left(Z_{i}-z\right)\right)^{2} k_{h}\left(W_{i}\right) k_{h}\left(Z_{i}-z\right) 1(W<0),
$$

$\widehat{A}_{+} g=\widehat{a}$ in

$$
\left(\widehat{a}, \widehat{b}_{0}, \widehat{b}_{1}\right)=\arg \min _{a_{0}, b_{0}, b_{1}} \sum_{i=1}^{n}\left(g\left(X_{i}\right)-a_{0}-b_{0} W-b_{1}\left(Z_{i}-z\right)\right)^{2} k_{h}\left(W_{i}\right) k_{h}\left(Z_{i}-z\right) 1(W \geq 0),
$$


and $\widehat{A}_{-} g=\widehat{a}$ in

$$
\left(\widehat{a}, \widehat{b}_{0}, \widehat{b}_{1}\right)=\arg \min _{a_{0}, b_{0}, b_{1}} \sum_{i=1}^{n}\left(g\left(X_{i}\right)-a_{0}-b_{0} W-b_{1}\left(Z_{i}-z\right)\right)^{2} k_{h}\left(W_{i}\right) k_{h}\left(Z_{i}-z\right) 1(W<0) .
$$

We can follow Theorem 2 in Blundell, Chen and Kristensen (2007) to obtain the rates for $\widehat{g}_{n}$,

$$
\left\|\widehat{g}_{n}-g\right\|=O_{P}\left(k_{n}^{-r}+\tau_{n} \times \sqrt{\frac{k_{n}}{n}}\right),
$$

provided we show that

$$
\|\widehat{m}-m\|=O_{P}\left(\sqrt{\frac{k_{n}}{n}}\right)
$$

and

$$
\sup _{g \in \mathcal{G}_{n}}\left\|(\widehat{A}-A) g\left(Z_{i}\right)\right\|=O_{P}\left(\sqrt{\frac{k_{n}}{n}}\right) ;
$$

see Claim 2 in p. 1658 of Blundell, Chen and Kristensen (2007). But Theorem 6 in Masry (1996) shows that

$$
\|\widehat{m}-m\|=O_{P}\left(d_{n}\right)
$$

where $d_{n}=\left(\log n / n h^{2}\right)^{1 / 2}+h^{4}$. Combining standard empirical processes arguments with the results of Masry (1996), we similarly obtain

$$
\sup _{g \in \mathcal{G}_{n}}\left\|(\widehat{A}-A) g\left(Z_{i}\right)\right\|=O_{P}\left(d_{n}\right) .
$$

Therefore, we require rates on the bandwidth $h$ so that

$$
d_{n}=O_{P}\left(\sqrt{\frac{k_{n}}{n}}\right) .
$$

This provides some flexibility in how to choose the bandwidth $h$. Higher order polynomial estimation improves the bias of the first step estimates, and leads to wider set of possible bandwidths. The reader is referred to Blundell, Chen and Kristensen (2007) and Masry (1996) for discussion on rates permitted for $k_{n}$ and $h_{n} \equiv h$ to obtain the desired rates for $\widehat{g}_{n}-g$ under different scenarios on the rate for the measure of ill-posedness. 


\section{References}

Ai, C., And X. Chen (2003): "Efficient Estimation of Models with Conditional Moment Restrictions Containing Unknown Functions," Econometrica, 71(6), 1795-1843.

Almond, D., And J. Currie (2011): "Killing Me Softly: The Fetal Origins Hypothesis," The Journal of Economic Perspectives, 25(3), 153-172.

Andrews, D. (2011): "Examples of L2-Complete and Boundedly-Complete Distributions," Cowles Foundation for Research in Economics.

Angrist, J. D., And A. B. Krueger (1991): "Does Compulsory School Attendance Affect Schooling and Earnings?," The Quarterly Journal of Economics, 106(4), 979-1014.

Angrist, J. D., And W. N. Evans (1998): "Children and Their Parents' Labor Supply: Evidence from Exogenous Variation in Family Size," American Economic Review, 88, 450-477.

Angrist, J., Graddy, K. and G. Imbens (2000): "The Interpretation of Instrumental Variables Estimators in Simultaneous Equations Models with an Application to the Demand for Fish," The Review of Economic Studies, 67, 499-527.

Blundell, R., X. Chen, and D. Kristensen (2007): "Semi-nonparametric IV Estimation of Shapeinvariant Engel Curves, " Econometrica, 75, 1613-1670.

Brollo, F., T. Nannicini, R. Perotti and G. Tabellini (2013): "The Political Resource Curse" American Economic Review, 103(5): 1759-96.

Calonico, S., Cattaneo, M.D. and Titiunik, R. (2014): "Robust Nonparametric Confidence Intervals for Regression-Discontinuity Designs", Econometrica, 82, 2295-2326.

CARD, D. (1995): "The Wage Curve: A Review," Journal of Economic Literature, vol. 33(2), 285-299.

Carpenter, C. and Dobkin, C. (2009): "The Effect of Alcohol Access on Consumption and Mortality: Regression Discontinuity Evidence from the Minimum Drinking Age", American Economic Journal: Applied Economics, Vol. 1, Issue 1, pp. 164-82.

Carrasco, M., J. P. Florens, and E. Renault (2006): "Linear Inverse Problem in Strucutral Econometrics Estimation Based on Spectral Decomposition and Regularization," in Handbook of Econometrics, vol. 6, ed. by J. J. Heckman and E. E. Leamer. Amsterdam: North-Holland, 56335751.

Chay, K., and M. Greenstone (2005): "Does Air Quality Matter? Evidence from the Housing Market," Journal of Political Economy, 113(2), 376-424.

Chen, X. (2007): "Large sample sieve estimation of semi-nonparametric models," in Handbook of Econometrics (J. J. Heckman and E. E. Leamer, eds.) volume 6, 5549-5632. Elsevier, Amsterdam. 
Chen, X., And D. Pouzo (2012): "Estimation of Nonparametric Conditional Moment Models with Possibly Nonsmooth Generalized Residuals," Econometrica, 80(1), 277-321.

Chesher, A. (2003): "Identification in Nonseparable Models," Econometrica, 71, 1405-1441.

Darolles, S., Y. Fan, J. P. Florens, and E. Renault (2011): "Nonparametric Instrumental Regression," Econometrica, 79(5), 1541-1565.

D'Haultfoeuille, X. (2011): "On the Completeness Condition in Nonparametric Instrumental Problems," Econometric Theory, 1, 1-12.

D'Haultfoeuille, X. And P. Fevrier (2014): "Identification of Nonseparable Models with Endogeneity and Discrete Instruments," Econometrica, forthcoming.

D'Haultfoeuille, X., Hoderlein, S. And Y. Sasaki (2013): "Nonlinear Difference-in-Differences in Repeated Cross Sections with Continuous Treatments", Boston College Working Paper wp839.

DiNardo, J., And D. S. LeE. (2011): "Program Evaluation and Research Designs," In Handbook of Labor Economics, ed. O. Ashenfelter and D. Card, vol. 4A, 463-536. Elsevier Science B.V.

Dunker, F., Florens, J-P., Hohage, T., Johannes, J. And Mammen, E. (2014): "Iterative Estimation of Solutions to Noisy Nonlinear Operator Equations in Nonparametric Instrumental Regression", Journal of Econometrics, 178, 444-455.

Fan, J., And Gijbels, I. (1996): Local Polynomial Modelling and its Applications. Chapman \& Hall, London.

Frolich, M. (2007): "Nonparametric IV Estimation of Local Average Treatment Effects with Covariates," Journal of Econometrics, 139, 35-75.

Hall, P., And J. Horowitz (2005): "Nonparametric Methods for Inference in the Presence of Instrumental Variables," Annals of Statistics, 33, 2904-2929.

Hahn, J., Todd, P., and van der Klaauw, W. (1999): "Evaluating the Effect of an Antidiscrimination Law Using a Regression-Discontinuity Design." National Bureau of Economic Research Working Paper 7131.

Hahn, J., Todd, P., and van der KlaAuw, W. (2001): "Identification and Estimation of Treatment Effects with a Regression-Discontinuity Design," Econometrica, 69, 201-09.

Horowitz, J. (2007): "Asymptotic normality of a nonparametric instrumental variables estimator," International Economic Review, 48, 1329-1349.

Horowitz, J. (2011): "Applied Nonparametric Instrumental Variables Estimation," Econometrica, $79(2), 347-394$. 
Imbens, G., And J. Angrist (1994): "Identification and Estimation of Local Average Treatment Effects," Econometrica, 61, 2, 467-476.

Imbens, G. W. And T. Lemieux (2008): "Regression Discontinuity Designs: A Guide to Practice." Journal of Econometrics, 142(2): 615-35.

KASY M. (2009): "Semiparametrically Efficient Estimation of Conditional Instrumental Variable Parameters," International Journal of Biostatistics, 5 (1).

KASY M. (2014): "Instrumental variables with unrestricted heterogeneity and continuous treatment," Review of Economic Studies, forthcoming.

Lee, D. S., And T. Lemieux (2010): "Regression Discontinuity Designs in Economics," Journal of Economic Literature 48, 281-355.

Lumley, J., C. Chamberlain, T. Dowswell, S. Oliver, L. Oakley, and L. Watson (2009): "Interventions for Promoting Smoking Cessation During Pregnancy (Cochrane Review)," The Cochrane Library, 8(3).

MAsRy, E. (1996): "Multivariate local polynomial regression for time series: uniform strong consistency and rates," Journal of Time Series Analysis, 17, 571-599.

Masten, M. And Torgovitsky, A. (2014): "Instrumental Variables Estimation of a Generalized Correlated Random Coefficients Model," CEMMAP working paper CWP02/14.

Matzkin, R.L. (2003): "Nonparametric Estimation of Nonadditive Random Functions," Econometrica, 71, 1339-13785.

Newey, W. K., And J. Powell (2003): "Instrumental Variables Estimation for Nonparametric Models," Econometrica, 71, 1565-1578.

SAntos, A. (2012): "Inference in Nonparametric Instrumental Variables with Partial Identification," Econometrica, 80, 213-275.

Severini, T. A., And G. Tripathi (2006): "Some Identification Issues in Nonparametric Linear Models with Endogenous Regressors," Econometric Theorey, 22(2), 258-278.

Severini, T. A., and G. Tripathi (2012): "Efficency Bounds for Estimating Linear Functionals of Nonparametric Regression Models with Endogenous Regressors," Journal of Econometrics, 170(2), 491-498.

Torgovitsky, A. (2014): "Identification of Nonseparable Models using Instruments with Small Support," Econometrica, forthcoming.

VAN Der KlaAuw, W. (2008): "Regression-Discontinuity Analysis: A Survey of Recent Developments in Economics." Labour, 22(2): 219-45. 